\title{
Neurons in a Forebrain Nucleus Required for Vocal Plasticity Rapidly Switch between Precise Firing and Variable Bursting Depending on Social Context
}

\author{
Mimi H. Kao, ${ }^{1}$ Brian D. Wright, ${ }^{1,2 \dagger}$ and Allison J. Doupe ${ }^{1,2}$ \\ ${ }^{1}$ Keck Center for Integrative Neuroscience, Departments of Physiology and Psychiatry, and ${ }^{2}$ Sloan-Swartz Center for Theoretical Neurobiology, University \\ of California, San Francisco, San Francisco, California 94143-0444
}

\begin{abstract}
Song is a learned vocal behavior influenced by social interactions. Prior work has suggested that the anterior forebrain pathway (AFP), a specialized pallial- basal ganglia circuit critical for vocal plasticity, mediates the influence of social signals on song. Here, we investigate the signals the AFP sends to song motor areas and their dependence on social context by characterizing singing-related activity of single neurons in the AFP output nucleus LMAN (lateral magnocellular nucleus of the anterior nidopallium). We show that interaction with females causes marked, real-time changes in firing properties of individual LMAN neurons. When males sing to females ("directed"), LMAN neurons exhibit reliable firing of single spikes precisely locked to song. In contrast, when males sing alone ("undirected"), the same LMAN neurons exhibit prominent burst firing and trial-by-trial variability. Burst structure and timing vary substantially across repeated undirected trials. Despite context-dependent differences in firing statistics, the average pattern of song-locked firing for an individual neuron is similar across behavioral contexts, suggesting a common underlying signal. Different LMAN neurons in the same bird, however, exhibit distinct firing patterns, suggesting that subsets of neurons jointly encode song features. Together, our findings demonstrate that behavioral interactions reversibly transform the signaling mode of LMAN neurons. Such changes may contribute to rapid switching of motor activity between variable and precise states. More generally, our results suggest that pallial-basal ganglia circuits contribute to motor learning and production through multiple mechanisms: patterned signals could guide changes in motor output while state-dependent variability could subserve motor exploration.
\end{abstract}

Key words: basal ganglia; vocal learning; burst firing; spike count variability; zebra finch; LMAN

\section{Introduction}

Song is a learned motor skill shaped by social experience. Interactions with conspecifics can influence which song models are memorized and which are retained during sensorimotor learning (West and King, 1988; Nelson and Marler, 1994). Social factors also influence adult song production. For example, male zebra finches alter the tempo and degree of variability in their songs to make them more attractive to females (Sossinka and Böhner, 1980; Kao et al., 2005; Cooper and Goller, 2006; Woolley and Doupe, 2008). Several findings suggest that a specialized pallialbasal ganglia circuit, known as the anterior forebrain pathway (AFP) (see Fig. $1 A$ ), is an important source of context-dependent

Received May 18, 2008; revised Aug. 29, 2008; accepted 0ct. 16, 2008.

This work was supported by a Howard Hughes Medical Institute Predoctoral Fellowship (M.H.K.), the Swartz Foundation (B.D.W.), the MacArthur Foundation, the Steven and Michele Kirsch Foundation, National Alliance for Research on Schizophrenia and Depression and the Grable Foundation, the Sandler Family Supporting Foundation, and National Institutes of Health [Grants MH55987 and MH077970 (A.J.D.)]. We thank Adria Arteseros and Lazlo Bocksai for technical assistance and Steve Lisberger, Michael Stryker, and Sarah C. Woolley for thoughtful comments on this manuscript. We would also like to specially acknowledge the tremendous intellectual contribution of our co-author Dr. Brian D. Wright, who passed away on Jan. 10, 2008.

${ }^{\dagger}$ Deceased, Jan. 10, 2008.

Correspondence should be addressed to Mimi H. Kao, Department of Physiology, Box 0444, 513 Parnassus Avenue, University of California, San Francisco, San Francisco, CA 94143-0444. E-mail: mimi@phy.ucsf.edu. DOI:10.1523/JNEUROSCI.2250-08.2008

Copyright $\odot 2008$ Society for Neuroscience ～0270-6474/08/2813232-16\$15.00/0 song variation. First, AFP activity is modulated by social context. In both the striatopallidal region Area X and the AFP output nucleus LMAN (lateral magnocellular nucleus of the anterior nidopallium), the immediate early gene (IEG) egr-1 is induced in more neurons when males sing alone ("undirected") than when they sing to females ("directed") (Jarvis et al., 1998). Multiunit activity in Area X and LMAN is also greater and more variable across renditions during undirected singing compared with during directed singing (Hessler and Doupe, 1999a). Second, lesions or inactivation of LMAN eliminate song variability and plasticity (Bottjer et al., 1984; Scharff and Nottebohm, 1991; Brainard and Doupe, 2000; Kao et al., 2005; Olveczky et al., 2005; Kao and Brainard, 2006).

These results raise questions regarding the nature of the social context-dependent changes in AFP activity. IEG and multiunit recording data suggest that an additional population of LMAN neurons becomes active during undirected song. Alternatively, greater IEG expression and neural activity during undirected song could derive from changes in firing properties of individual LMAN neurons. Distinguishing these possibilities requires examination of single unit activity in both social states. Single unit recordings can also provide a mechanistic understanding of LMAN's contribution to vocal production and plasticity. For instance, neural firing could be bursting or tonic, patterned or 
random, homogenous or diverse across the population, and the particular firing properties would inform hypotheses about LMAN's function. Finally, since context dependence of neural activity is a common feature of cortical-basal ganglia circuits (Hikosaka et al., 2006), studies of single LMAN neurons in different contexts are relevant for understanding information processing in mammalian basal ganglia circuits.

By recording many trials of single neuron activity during directed and undirected singing, we show for the first time that individual LMAN neurons rapidly switch between precisely locked firing of single spikes and highly variable firing dominated by bursts. Variation in burst structure and timing contribute to greater trial-by-trial variability during undirected singing. Despite marked context-dependent differences in firing statistics, the average pattern of song-locked firing is surprisingly similar across conditions, suggesting that this pattern reflects a common signal, with additional variability when birds are alone. Finally, by recording multiple single units in the same bird, we show that different LMAN neurons exhibit distinct song-locked firing patterns. Together, our findings suggest that LMAN uses multiple mechanisms for transmitting information about song: patterned signals could guide systematic changes, and marked bursting and trial-by-trial variability could subserve vocal exploration. In mammalian systems, different cortical-basal ganglia subcircuits are thought be active during motor learning versus during the execution of well learned skills (Costa, 2007). Here, we show in addition that individual neurons within a single subcircuit can dramatically shift their activity between precise "performance" and variable "exploratory" states.

\section{Materials and Methods \\ Subjects}

Nine adult ( $>125$ posthatch days) male zebra finches (Taeniopygia guttata) raised in our colony were used in this study. For all experiments, birds were isolated in a small cage inside a sound-attenuating chamber (Acoustic Systems). All procedures were performed in accordance with protocols approved by the University of California, San Francisco (UCSF) Institutional Animal Care and Use Committee.

\section{Surgical procedures}

Surgery for the implantation of a small microdrive carrying tungsten electrodes was performed as described previously (Hessler and Doupe, 1999a). Briefly, birds were deprived of food and water for $1 \mathrm{~h}$ and then anesthetized with an intramuscular injection of $30-40 \mu \mathrm{l}$ of equithesin ( $0.85 \mathrm{~g}$ of chloral hydrate, $0.21 \mathrm{~g}$ of pentobarbital, $0.42 \mathrm{~g}$ of $\mathrm{MgSO}_{4}, 2.2 \mathrm{ml}$ of $100 \%$ ethanol, and $8.6 \mathrm{ml}$ of propylene glycol to a total volume of $20 \mathrm{ml}$ with water). A lightweight microdrive [UCSF and California Institute of Technology (Caltech) Machine Shops] carrying two glass-coated tungsten electrodes spaced $\sim 300-600 \mu \mathrm{m}$ apart (2-5 M ; A. Ainsworth, Northhamptonshire, UK) (Merrill and Ainsworth, 1972) was positioned stereotaxically such that the electrode tips were $\sim 700 \mu \mathrm{m}$ dorsal to LMAN (5.0 $\mathrm{mm}$ anterior and $1.7 \mathrm{~mm}$ lateral to the posterior border of the divergence of the central sinus). A reference ground electrode (uninsulated tungsten electrode; A-M Systems) was implanted in the contralateral hemisphere such that its tip was located within $2 \mathrm{~mm}$ of the targeted nucleus. The microdrive and connector socket (FHC) were secured to the skull with epoxy (Devcon) and dental cement (Dentsply), and a protective cap was fixed around the microdrive. All recordings were made from the right hemisphere.

\section{Electrophysiology and data collection}

During each experimental session, one end of a flexible lead terminating in a small operational amplifier circuit (Texas Instruments) was connected to the socket on the bird's head, and the other end was connected to a mercury commutator (Caltech Machine Shop, Pasadena, CA). Electrodes were lowered to sites in LMAN where action potentials of single or multiple units could be clearly differentiated from the background neural activity. Spike amplitudes ranged from $500 \mu \mathrm{V}$ to $>10 \mathrm{mV}$, peak to peak. The neural activity signal passed through the commutator to a differential amplifier (A-M Systems) and was filtered between $300 \mathrm{~Hz}$ and 10 $\mathrm{kHz}$. The acoustic signal was recorded by a small microphone (Countryman) located above the birdcage and bandpass filtered between $200 \mathrm{~Hz}$ and $9 \mathrm{kHz}$ (Krohn-hite). The bird's behavior was monitored and recorded via a video camera inside the sound-attenuating chamber. Custom-written acquisition software (A. Leonardo, Caltech, Pasadena, CA and C. Roddey, UCSF, San Francisco, CA) recorded acoustic and neural signals.

Extracellular recordings were made at intervals of $1 \mathrm{~h}$ to several weeks, over a period of weeks to months. Typically, at the end of the recording session(s) on each day, the electrodes were retracted to a position above LMAN. Neural activity was recorded during nonsinging and singing periods. Spontaneous activity was recorded during periods when the bird was silent; such periods were required to precede or follow any vocalization by at least $3 \mathrm{~s}$ (Hessler and Doupe, 1999a). Recordings were made in two behavioral contexts: undirected, when the male was isolated in the sound-attenuating chamber, and directed, when the male sang to a female. To elicit directed song, one or more female zebra finches was presented in a separate cage to the male being recorded. The recorded bird usually moved to the edge of its cage and sang while facing the female(s). Each female presentation lasted for $\leq 2 \mathrm{~min}$, regardless of whether or not the male sang, and songs were classified as directed only when the male bird faced the female(s). Directed and undirected songs were interleaved during experimental sessions, which lasted between 30 min and several hours depending on singing frequency and the stability of the recording site. In some experiments, recordings were only possible in one of the two behavioral conditions.

At the conclusion of experiments in each bird, small electrolytic lesions (30 $\mu \mathrm{A}$ for $10 \mathrm{~s}$ ) were made at a subset of previously recorded sites. Animals were deeply anesthetized with metofane (Schering-Plough) and transcardially perfused with $0.9 \%$ saline, followed by $3.7 \%$ formaldehyde in $0.025 \mathrm{M}$ phosphate buffer. Electrode tracks and lesions were localized in $40 \mu \mathrm{m}$ Nissl-stained sections. Locations of all recorded sites were confirmed by their position relative to the depth of marker lesions (supplemental Fig. 1, available at www.jneurosci.org as supplemental material).

\section{Data analysis}

Neural activity was analyzed off-line, and large single units with a signalto-noise ratio $>3: 1$ were discriminated using Bayesian spike-sorting techniques (Lewicki, 1994) (code originally written by M. Lewicki, Caltech, Pasadena, CA; algorithm corrections and improvements by B. Wright, UCSF, San Francisco, CA; user interface improvements by M. Kvale and B. Wright, UCSF, San Francisco, CA). We verified the isolation of single units by visual inspection of the waveforms and by the presence of a refractory period in the interspike interval (ISI) histogram (refractory period $\leq 0.7 \mathrm{~ms} ;<1 \%$ ISI violations for each cell; $n=28$ units in 9 birds). Only one unit was isolated from each electrode; a second unit recorded by the same electrode was never discriminated either because it was not present or because we were less confident of its isolation. LMAN neurons in this study $(n=28)$ comprise a homogenous population with large spike amplitudes and low spontaneous firing rates, consistent with previous studies of putative and identified LMAN projection neurons in juvenile and adult zebra finches (Leonardo, 2004; Olveczky et al., 2005). To date, there have been no reports of LMAN interneurons recorded extracellularly, and previous intracellular studies have demonstrated the great difficulty of recording from LMAN interneurons, both in anesthetized adult birds and in a slice preparation (Livingston and Mooney, 1997; Rosen and Mooney, 2000).

\section{Alignment of song and neural data}

Zebra finch song can be classified into three levels of organization: "syllables," which are individual song elements separated by silent intervals at least $5 \mathrm{~ms}$ in duration; "motifs," which are stereotyped sequences of syllables; and "bouts" of song, which are defined as periods of singing separated by silent intervals at least $2 \mathrm{~s}$ in duration (Sossinka and Böhner, 1980). In our experimental design, which aimed to quantitatively assess 
trial-by-trial variability in the activity of single units in two different conditions, we recorded and analyzed the singing-related activity of neurons during numerous renditions of the song motif ("trials"), in contrast to previous single unit studies in which many recordings were brief (Leonardo, 2004). The mean number of undirected trials was $58 \pm 37$ (SD; range: 13-157), and the mean number of directed trials was $32 \pm 16$ (range: 12-59). For the 14 neurons recorded in both conditions, the mean number of trials was $71 \pm 36$ and $34 \pm 18$, respectively.

We analyzed motif-related neural activity beginning $40 \mathrm{~ms}$ before motif onset and ending $40 \mathrm{~ms}$ before motif offset, based on our estimate of the premotor latency between LMAN activity and song output from previous microstimulation experiments. Brief electrical stimulation in LMAN during singing can induce changes in syllable structure with a mean latency of $\sim 50 \mathrm{~ms}$ (Kao et al., 2005). For all analyses, we also tested a range of latencies (40-120 ms before motif onset), and none of our findings were dependent on the particular choice of latency.

The length of syllables and intersyllable intervals varies from one rendition to the next, resulting in noise in the structure of spike trains if they are aligned only at the onset of each song motif (Chi and Margoliash, 2001). To compensate for differences in the duration of syllables and intervals across trials, we performed a piecewise linear time warp of each syllable and interval so that all of the motifs and spike trains were on a common-aligned time axis. The alignment algorithm proceeded as follows. First, syllable boundaries were determined using an automated amplitude-based segmentation algorithm (i.e., threshold crossing of the acoustic power) (Kao et al., 2005; Leonardo and Fee, 2005). Consistent with Hessler and Doupe (1999a), we did not observe any systematic differences in song amplitude across behavioral conditions. Next, we manually labeled the syllables ("a," "b," "c," etc.), and measured the onset time, offset time, and duration for each syllable and interval. We chose the song motif with the median duration during the recording session to use as a reference motif for aligning the data. For every rendition of the motif, or trial, each syllable was linearly stretched or compressed to match the duration of the corresponding syllable in the reference motif. For example, every rendition of the syllable a was stretched or compressed to match the duration of the syllable a in the reference motif. Syllables were then aligned at the onset of the reference syllables. The same procedure was applied independently to the intervals. Next, the spike train was projected onto the time-warped axis of the corresponding syllable or interval. Thus, the duration of each trial was fixed to the duration of the reference motif. By stretching or compressing the syllables and intervals in different trials and the associated neural activity to a common reference and aligning their onsets, this algorithm reduced the variability in the temporal registry of the spike trains. To quantify this reduction in variability, for each neuron, we computed the correlation coefficient between the instantaneous firing rates (smoothed with a Gaussian filter; SD $=2 \mathrm{~ms}$; see below) for all pairs of trials in a particular behavioral condition before and after implementing piecewise linear time warping. The mean difference between the average correlation coefficient of the raw data and the time-warped data ranged from 0 to 0.12 .

For analyses of singing-related activity across multiple neurons during directed singing, the same algorithm was applied to align the motifs and spike trains, but the reference motif was the motif with the median duration across all recording sessions in each bird $(n=2-8$ recording sessions per bird in 5 birds).

To characterize LMAN activity preceding songs, we analyzed cases in which the bird was quiet for at least $2 \mathrm{~s}$ before initiation of a song bout (mean number of bouts: $7.2 \pm 1.1$ (SEM) in the undirected condition and $3.6 \pm 0.5$ in the directed condition). To characterize postsinging activity, we limited our analysis to songs that were followed by at least $3 \mathrm{~s}$ of silence ( $13.32 \pm 1.8$ bouts in the undirected condition and $2.89 \pm 0.4$ bouts in the directed condition). A longer interval of silence after song was chosen because Hessler and Doupe (1999b) showed that LMAN activity could remain suppressed for up to two seconds following song termination. Firing rates before and after song were normalized by the mean firing rate during nonsinging periods.

\section{Burst classification}

Spike trains of LMAN neurons consisted of bursts and isolated spikes. Many studies have identified burst events using an ISI criterion determined from the bimodal structure of ISI histograms (e.g., identifying the first trough following the peak of the distribution or identifying peaks in the autocorrelation function; Turner et al., 1996; Bastian and Nguyenkim, 2001). In this study, we found that during undirected singing, the ISI distributions of LMAN neurons typically exhibited a prominent peak at $\sim 2-3 \mathrm{~ms}$, followed by a steep decrease between 5 and $10 \mathrm{~ms}$ and a lower, broad plateau at longer intervals, similar to that of bursting neurons in other systems (see Fig. $1 E$; supplemental Fig. 2, available at www. jneurosci.org as supplemental material). Across all neurons, the average mode of the ISI distributions was $2.3 \pm 0.6 \mathrm{~ms}(\mathrm{SD})$ during undirected singing. On the basis of these observations, we defined bursts as groups of two or more action potentials separated by $\leq 5 \mathrm{~ms}$ (instantaneous firing rate $\geq 200 \mathrm{~Hz}$ ). The "burst fraction" of a spike train was defined as the fraction of all spikes that were contained in bursts. A less stringent criterion for bursts (ISI $\leq 10 \mathrm{~ms}$ ) did not qualitatively change any of the results reported.

\section{Analysis of trial-by-trial variability}

Pairwise correlation coefficients between trials. To quantify trial-by-trial variability on short time scales, we computed the correlation coefficient between the instantaneous firing rates for all pairs of trials. For a given trial, $i$, we estimated the instantaneous firing rate, $r_{i}(t)$, by smoothing the spike trains with a Gaussian filter of different SDs $(2,4,10$, and $20 \mathrm{~ms})$ and then subtracting the time-averaged firing rate for that trial, $\bar{r}_{i}$. The average correlation coefficient (CC) of the instantaneous firing rates for pairs of trials was as follows:

$$
\mathrm{CC}=\frac{1}{N_{\text {pairs }}} \sum_{j>i}^{N_{\text {trials }}} \mathrm{CC}_{i j},
$$

where

$$
\mathrm{CC}_{i j}=\frac{\left\langle\hat{r}_{i}(t) \hat{r}_{j}(t)\right\rangle_{t}}{\sqrt{\left\langle\hat{r}_{i}(t)^{2}\right\rangle_{t}\left\langle\hat{r}_{j}(t)^{2}\right\rangle_{t}}}
$$

and

$$
\hat{r}_{i}(t)=r_{i}(t)-\bar{r}_{i} .
$$

To compare our results to a measure of random correlation, all spike trains were also randomly time shifted ( $\pm 100-500$ ms; "shuffled" spike trains), and correlations were calculated for all pairs of shuffled spike trains. None of our findings were sensitive to the width of the filter used. Similar results were found when the instantaneous firing rate, $r_{i}(t)$, at each time point $t$ for trial $i$ was defined as the inverse of the enclosed interspike interval, as in the study by Leonardo and Fee (2005):

$$
r_{i}(t)=\frac{1}{t_{k+1}-t_{k}} \text { for } t_{k} \leq t<t_{k+1},
$$

and were used for comparison with the results of Olveczky et al. (2005).

Fano factor. As a second measure of trial-by-trial variability in neural activity, we computed the Fano factor $(F)$, which is defined as the variance of the spike count within a window of size $T$ divided by the mean spike count: $F_{(T, t)}=\sigma^{2} / \mu$, where $t$ is the time in the song where the onset of the window begins. Thus, $F$ is a function of both the counting window $T$ as well as the time in the motif, $t$. In all cases, we analyzed the singingrelated activity associated with a motif (see above). We counted the number of spikes in a sliding window of width $T$ ( 30 or $100 \mathrm{~ms}$ ) for each motif rendition, or trial, evaluated at times $t$ separated by $1 \mathrm{~ms}$. The mean and variance of the spike count were computed for each window $T$ across all trials in a particular behavioral condition. For a Poisson process, $F_{(T, t)}=$ 1 for any window $T$ at all times $t$, regardless of the fact that the firing rate varies with time within each trial. For comparison with spontaneous activity when the bird was silent, we divided the nonsinging periods into $1 \mathrm{~s}$ segments and computed the Fano factor in a sliding window $(T=30$ or $100 \mathrm{~ms}$ ) across all segments. 
Analysis of spike timing precision

To measure the maximal precision of spike timing across trials, we used a histogram-based measure similar to that of Mainen and Sejnowski (1995). For each LMAN neuron, the time-warped spike trains were binned to $1 \mathrm{~ms}$ resolution, and the binned data were compiled over all trials into a histogram that represents the probability of firing during the motif as a function of time. The spike time histogram was smoothed with a Hanning filter of different SDs (2, 4, and $10 \mathrm{~ms})$, and a threshold (mean firing rate $+4 \mathrm{SD}$ ) was used to identify the boundaries of significant elevations in firing rate, or "spike events." Once event boundaries were determined, subsequent analysis was performed on the raw spike times and no longer depended on the choice of smoothing. We excluded from further analysis any event that did not contain spikes in at least $50 \%$ of the trials. This reliability criterion was intended to eliminate events that may have been caused by factors unrelated to the song. For each reliable event, we computed the SD of the times of the first spike in the event across all trials. Spike timing precision, or "temporal jitter," was defined as the average of this measure over all events during the song motif. This procedure produced an estimate of the maximal degree of precision of LMAN spikes during singing. Spike timing precision was similar across all of the filter widths used as well as when the data were binned without smoothing. Similar results were obtained when the firing rate threshold was lowered (mean firing rate $+3 \mathrm{SD}$; data not shown) to include more spike events in our analysis.

\section{Analysis of similarity in mean firing patterns}

To quantify the degree of similarity in the firing pattern of a single LMAN neuron across behavioral contexts, we calculated the average activity pattern during directed singing and the average activity pattern during undirected singing and then computed the correlation coefficient between these two patterns (after subtraction of the time-averaged firing rate). The mean activity patterns were calculated by binning the spike count in each trial to $1 \mathrm{~ms}$ resolution, convolving this with a $10 \mathrm{~ms}$ Hanning window, and then averaging the smoothed spike counts across all trials. For such within-neuron analyses across behavioral contexts, the maximum correlation coefficient between mean activity patterns occurred at a time delay of $0 \mathrm{~ms}$.

To assess the similarity of firing patterns across multiple LMAN neurons in the same bird, we calculated the average activity patterns of different neurons during directed singing, and computed the correlation coefficients between the mean activity patterns of all pairs of neurons. When computing the correlation coefficients across different LMAN neurons in the same bird, we allowed for a time shift in the mean firing patterns (between $-40 \mathrm{~ms}$ and $+40 \mathrm{~ms}$, in $5 \mathrm{~ms}$ steps) to take into account the fact that the activity of different neurons could have different delays relative to the song output, and searched for the maximum correlation coefficient.

\section{Results \\ Social context modulates the firing statistics of LMAN neurons}

Extracellular signals were recorded from 28 single units in LMAN of nine freely behaving adult male zebra finches. We found that social context strongly modulates the firing properties of single LMAN neurons. Figure $1, B-F$, illustrates a representative experiment in which a male sang many renditions of a stereotyped sequence of syllables, or "motif," both in the presence of a female and when it was alone. Directed and undirected songs were interleaved throughout the experiment, and the firing properties of the neuron changed by the time of the first motif produced after a female was presented or removed (Fig. $1 B, E$; supplemental Fig. 3, available at www.jneurosci.org as supplemental material). Across repeated renditions of the motif, or trials, the firing rate for this representative LMAN neuron was significantly greater during undirected song than during directed song [mean \pm SD: $32.6 \pm 5.3$ spikes per second $(\mathrm{Hz})$ vs $23.9 \pm 4.8 \mathrm{~Hz} ; p<0.0001$; Mann-Whitney $U$ test (M-W test)]. Across the population of
LMAN neurons that we recorded, the average firing rate increased significantly from $8.9 \pm 4.7 \mathrm{~Hz}$ during nonsinging periods to $21.1 \pm 9.63 \mathrm{~Hz}$ during directed singing $(n=23 ; p<$ $0.0001)$ and $34.9 \pm 8.5 \mathrm{~Hz}$ during undirected singing $(n=19)$ (Fig. 2A).

Higher firing rates during undirected singing were due, in large part, to the appearance of numerous bursts of action potentials, which were much less frequent during directed song and during nonsinging periods. Context-dependent differences in bursting are apparent in the ISI histograms (Fig. $1 E$, right panels). During undirected singing, the ISI distributions consisted of the following two phases: a prominent peak at short intervals $(\sim 2-3 \mathrm{~ms})$, which dropped off steeply between 5 and $10 \mathrm{~ms}$, followed by a lower, broad plateau at longer intervals (supplemental Fig. 2, available at www.jneurosci.org as supplemental material). In contrast, during directed singing and during nonsinging periods, there was a paucity of short ISIs. Based on the ISI histograms, we defined bursts to be any group of two or more spikes that have intervals $\leq 5 \mathrm{~ms}$, and determined burst onsets and offsets using a threshold instantaneous firing rate of $200 \mathrm{~Hz}$ (see Materials and Methods). For the neuron in Figure 1, the fraction of spikes that occurred in bursts was substantially higher during undirected song than during directed song and during nonsinging periods (mean burst fraction $=56.1 \%, 4.6 \%$, and $3.7 \%$, respectively). Across the population of LMAN neurons, bursting was significantly greater during undirected song compared with during directed song (Fig. $2 \mathrm{~B}$ ) (mean burst fraction: $58.0 \pm 12.8 \%$ vs $9.2 \pm 7.8 \% ; p<0.0001 ; \mathrm{M}-\mathrm{W}$ test; range: $33.7-80.5 \%$ vs $0-24 \%$, respectively) and compared with nonsinging periods (Fig. $2 B)$ ( $p<0.0001$; paired sign test). Moreover, the social context-dependent difference in bursting was observed in every LMAN neuron that was recorded in both behavioral conditions (Fig. $2 B$, filled symbols) $(n=14$; lines connect data from the same neuron; $p=0.0001$; paired sign test). Similar results were observed when a less stringent criterion was used to define bursts (ISI $\leq 10 \mathrm{~ms}$, data not shown).

\section{Trial-by-trial variability in LMAN activity is greater during undirected song}

Trial-by-trial variability in the firing patterns of single LMAN neurons was also markedly altered by social context. We took advantage of the stereotyped acoustic features of zebra finch song and aligned the activity of each LMAN neuron to a common reference motif. This procedure resulted in an improvement in the alignment of the spike trains, especially in the directed condition (see Materials and Methods), indicating that some of the variability in the raw spike trains is related to the variable timing of syllable onsets and offsets from trial to trial. Using the motifaligned spike trains, we observed that the firing patterns of LMAN neurons were reliably locked to the song in the directed condition (Fig. $1 E$, top), but much less so in the undirected condition (Fig. $1 E$, middle).

To measure reproducibility in spike train patterns on short time scales, we first computed the correlation coefficients between the instantaneous firing rates (estimated by smoothing the spike trains with a Gaussian filter; see Materials and Methods) for all pairs of trials in a particular behavioral condition, and then averaged them for each LMAN neuron. This measure is sensitive to the jitter in the timing of the spikes across trials. After smoothing with a narrow filter, the correlation between two spike trains is high only if the timing of spikes, or pattern, is similar across trials (i.e., when the jitter is less than or equal to the filter width). Figure $3 A$ shows the average correlation coefficient ( \pm SEM) be- 


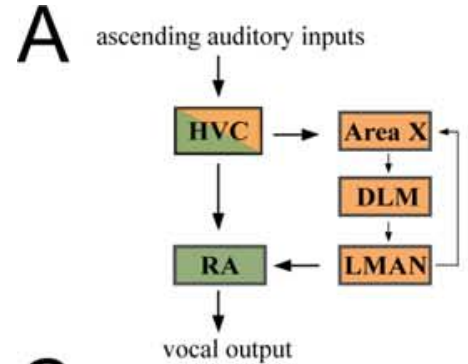

C
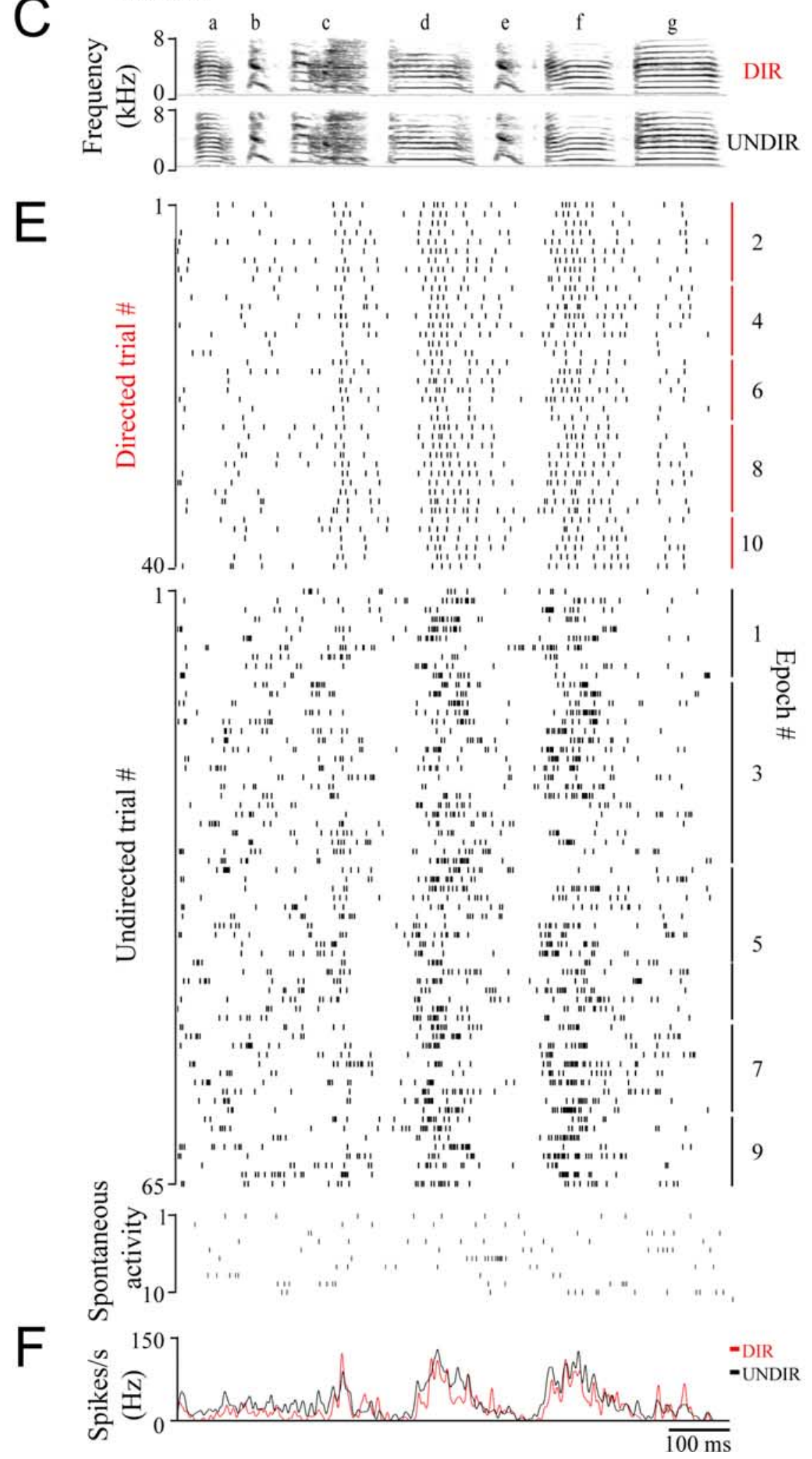

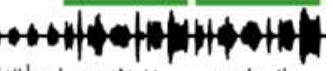

DIR

UNDIR

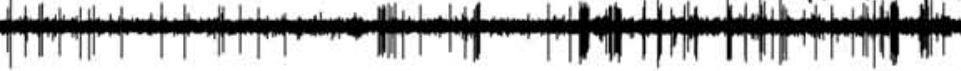

$1000 \mathrm{~ms}$
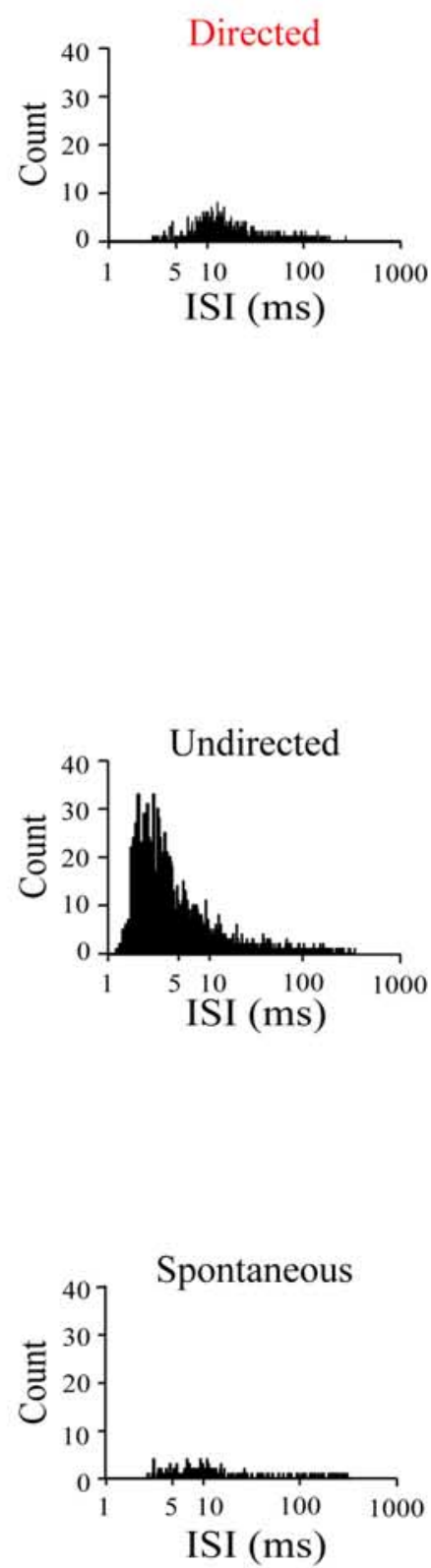
tween the smoothed spike trains (SD of the Gaussian filter $=10$ $\mathrm{ms}$ ) for neurons during directed singing versus during undirected singing. The diagonal line indicates equal correlation across the two social conditions. For each neuron with recordings in both conditions $(n=14)$, correlations in the spike trains across different renditions of the motif were greater during directed song than during undirected song $[p=0.0001$, paired sign test for comparisons across context; range of mean correlation coefficients (green diamonds): $0.12-0.87$ (directed) and 0.07-0.35 (undirected)]. Similarly, across the population of neurons with recordings in only one behavioral condition, greater reproducibility in the spike trains across repeated trials was observed during directed singing compared with during undirected singing (Fig. $3 B)(p=0.003, \mathrm{M}-\mathrm{W}$ test). We also compared the distribution of correlation coefficients with those calculated after the spike trains were randomly shifted in time (Fig. $3 A$, black circles) (see Materials and Methods). In both behavioral conditions, the distribution of correlation coefficients of each neuron was significantly different from that of the randomly shuffled spike trains $(p<0.0001, \mathrm{M}-\mathrm{W}$ test $)$, indicating that the activity of LMAN neurons was patterned with respect to song, even in the undirected condition.

As a second measure of trial-by-trial variability, we computed the Fano factor (ratio of the spike count variance to the mean spike count; $F$ ) in short time windows. Variability of neuronal spike trains is often characterized in reference to a Poisson process, in which each event occurs with a fixed probability that is independent of the occurrence of other events. For a Poisson process, $F$ has a value of 1 . If $F$ is $>1$, variance in the spike count distribution is high and reliability is low compared with the Poisson standard. Values of $F<1$ reflect increased reliability of firing and structure in spike trains, such as temporal correlations between spikes. For each trial, we computed spike counts in a sliding window ( 30 or $100 \mathrm{~ms}$ ) and then calculated the mean count and variance across trials for each neuron in a particular behav-

\section{$\leftarrow$}

Figure 1. Social context modulates the firing properties of single neurons in LMAN. $\boldsymbol{A}$, Schematic depicting the major functional divisions of the forebrain song control circuitry. The "motor pathway" (green) includes HVC (used as a proper name) and the robust nucleus of the arcopallium (RA), which innervates the hypoglossal motor neurons and respiratory motor neurons used for singing. The "anterior forebrain pathway" (AFP; orange) is necessary for vocal plasticity and consists of the basal ganglia homolog Area $X$, the medial nucleus of the dorsolateral thalamus (DLM), and the lateral magnocellular nucleus of the anterior nidopallium (LMAN). $\boldsymbol{B}$, Example of singing-related activity in LMAN before and during two repetitions of a stereotyped sequence of syllables, or "motifs" (green bars), when a male (blueblue) sings to a female (directed song, DIR; top) or when it sings alone (undirected song, UNDIR; bottom). Amplitude oscillograms of sound recordings are plotted above the LMAN neural activity. All subsequent data in this figure are from the same experiment. $C$, Spectrograms (frequency vs time) of a representative song motif (abcdefg) during DIR and UNDIR. D, One hundred randomly selected action potential waveforms of an isolated LMAN unit sorted from this experiment are superimposed. $\boldsymbol{E}$, Raster plot of the singing-related activity of the LMAN neuron during 40 renditions of DIR (top) and 65 renditions of UNDIR (middle). Each line represents an action potential, and each row represents the singing-related activity during one rendition of the motif, or "trial." Epochs of DIR (red bars) and UNDIR (black bars) were interleaved, beginning with UNDIR (supplemental Fig. 3, available at www.jneurosci.org as supplemental material). Spontaneous activity of the same neuron when the male was alone is plotted below (bottom). Firing rate was greater during UNDIR (mean \pm $\mathrm{SD}=32.6 \pm 5.3 \mathrm{~Hz}$ ) compared with during DIR $(23.9 \pm 4.8 \mathrm{~Hz} ; p<0.0001$; Mann-Whitney $U$ test) and compared with spontaneous activity $(11.54 \pm 5.08 \mathrm{~Hz})$. Corresponding histograms of the ISIs (log scale) during singing and nonsinging conditions are plotted to the right of the rasters. The neuron frequently produced short bursts of action potentials during UNDIR (middle; note the peak from $\sim 2$ to $3 \mathrm{~ms}$ ) but only rarely during DIR (top) and during nonsinging periods (bottom). $\boldsymbol{F}$, The average pattern of activity was similar during DIR (red) and UNDIR (black; correlation coefficient $=0.78$ ), despite the greater trial-by-trial variability during UNDIR.
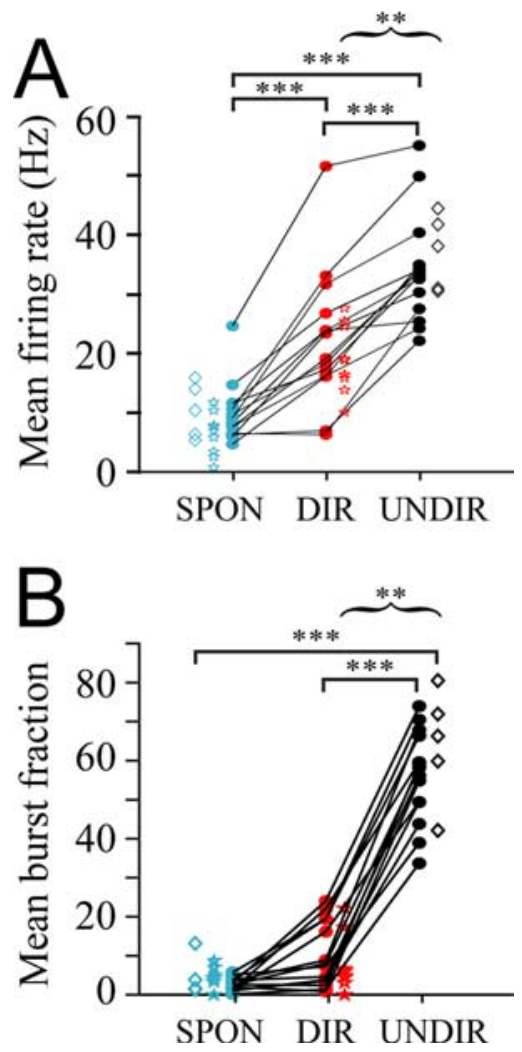

Figure 2. Firing rate and bursting are greater during UNDIR than during DIR. $\boldsymbol{A}$, Across the population of LMAN neurons, the mean firing rate increased significantly during singing DIR (red; $n=23$ neurons; $p<0.0001$, paired sign test) and during UNDIR (black; $n=19$ neurons; $p<0.0001$, paired sign test) compared with the spontaneous activity when birds were silent (SPON; cyan). Moreover, mean firing rates were significantly higher during UNDIR than during DIR ( $p<0.0001, M-W$ test). For the subset of neurons with recordings in both behavioral conditions (filled symbols; $n=14$ in seven birds; lines connect data from the same neuron), the mean firing rate was $32.4 \mathrm{~Hz}$ during UNDIR compared with $22.5 \mathrm{~Hz}$ during DIR ( $p=0.0001$, paired sign test). This context-dependent difference in firing rate was significant in 12 of 14 neurons when considered individually [ $p=0.0001$, Mann-Whitney $U$ test (M-W test)]. Open symbols denote data for neurons recorded either only during DIR ( $n=9$ in four birds) or only during UNDIR ( $n=5$ in two birds). Here, and in subsequent figures, data from neurons recorded in only one condition are not significantly different from the corresponding data for neurons recorded in both conditions. Triple asterisks denote $p<0.0005$; double asterisks denote $p<$ 0.005 ; and a single asterisk denotes $p<0.01$. Straight brackets indicate paired comparisons, and curly brackets indicate unpaired comparisons. Same conventions are used for all subsequent figures. $\boldsymbol{B}$, The fraction of spikes that occurred in bursts (instantaneous firing rate $\geq 200$ $\mathrm{Hz}$; "burst fraction") was significantly greater during UNDIR compared with the spontaneous activity when the bird was silent ( $p<0.0001$, paired sign test). LMAN neurons also fire more bursts of action potentials during UNDIR than during DIR. This context-dependent difference in bursting was present in every neuron with recordings in both behavioral conditions ( $n=14$; $p=0.0001$, paired sign test) as well as across the population of neurons recorded in only one condition ( $p=0.003, \mathrm{M}-\mathrm{W}$ test).

ioral condition. We found that $F$ varied as a function of time in the motif and was generally higher throughout the entire motif in the undirected condition compared with the directed condition. Figure 3, $C$ and $D$, illustrates the modulation of firing rate and spike count variability during song for two representative LMAN neurons. For these neurons, the time-averaged $F$ for all $30 \mathrm{~ms}$ windows was greater in the undirected condition than in the directed condition ( 1.82 vs 0.67 and 2.21 versus 0.81 , respectively) (Fig. $3 D$ ). Across the population of LMAN neurons recorded, spike count variability was significantly greater during undirected singing than during directed singing and during nonsinging periods (Fig. 3E). For neurons with recordings in both 
A

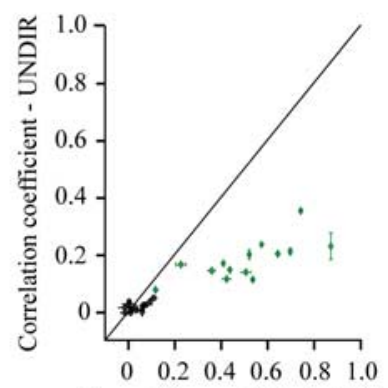

Correlation coefficient - DIR
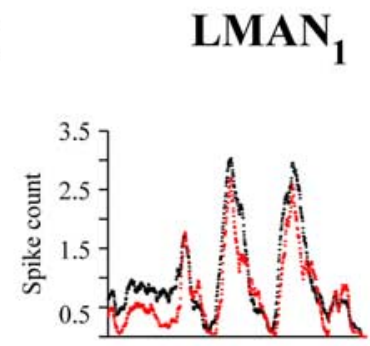

D
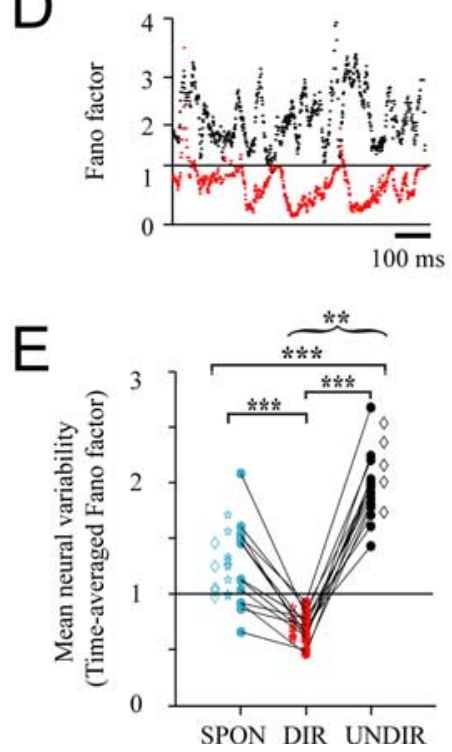

Figure 3. Variability in neural activity across repeated renditions of song is greater during UNDIR than during DIR. $A$, For each LMAN neuron, trial-by-trial variability in the firing pattern was quantified by computing the mean spike-train correlation coefficients ( \pm SEM) across all pairs of trials in a particular behavioral condition. The spike trains were smoothed with a Gaussian filter ( $S D=10 \mathrm{~ms})$. In 14 of 14 neurons in seven birds, correlations in the spike trains across different trials were significantly greater during DIR than during UNDIR (green diamonds; $p<$ $0.001, \mathrm{M}-\mathrm{W}$ test; average correlation ( $\pm S \mathrm{SD}$ ) across all neurons: $0.50 \pm 0.20$ and $0.18 \pm 0.07$, respectively). In both behavioral conditions, the distributions of correlation coefficients were also significantly different from that calculated after the spike trains were randomly shifted in time (black circles; $p<0.0001$; see Materials and Methods). $\boldsymbol{B}$, Mean spike-train correlations are plotted for neurons recorded in only one behavioral condition (open symbols). Correlations in the spike trains across repeated trials were significantly greater during DIR (red) than during UNDIR (black; $p=0.003 ; M-W$ test). For comparison, the mean spike-train correlation coefficients $( \pm S D)$ are plotted for the neurons with recordings in both behavioral conditions (filled symbols). C, Mean spike count (firing rate) in a $30 \mathrm{~ms}$ sliding window for two LMAN neurons from two birds (blueblue and b39614) during DIR (red) and UNDIR (black). Each point represents the spike count in a $30 \mathrm{~ms}$ window, computed at times separated by $1 \mathrm{~ms}$. Data on the left are from the neuron shown in Figure 1.D, Fano factor $(F)$ for the same data through the course of the motif. Data for a Poisson process would fall on the line $F=1$. Spike count variability was generally higher throughout the motif during UNDIR. $E$, The average $F$ across all $30 \mathrm{~ms}$ windows is plotted for individual neurons during nonsinging periods (SPON), DIR and UNDIR. Reliability social contexts, the mean time-averaged $F$ was 1.95 during undirected song, 0.69 during directed song, and 1.27 during nonsinging periods (Fig. $3 E$, filled symbols with lines connecting data from the same neuron) ( $p<0.0001$; paired sign tests). Similar results were obtained with $100 \mathrm{~ms}$ sliding windows (mean $F=$ $2.4,0.62$, and 1.87 , respectively).

To test whether context-dependent differences in spike count variability simply reflected the observed differences in firing rate between social contexts, we analyzed the variability of singingrelated activity as a function of firing rate (spike count). For each neuron, we compared the variance in spike count to the mean spike count in overlapping $100 \mathrm{~ms}$ windows throughout the song motif. Although this analysis does not take into account the temporal relationship between song and firing rate, it allows comparison of variability across behavioral conditions at any particular firing rate. Figure $4 A$ illustrates this analysis for the two representative LMAN neurons of Figure 3. During undirected song, across a range of mean counts (firing rates), the variance generally exceeded the mean spike count $(F>1)$. In contrast, during directed song, a majority of windows had variance below the mean spike count $(F<1)$. Across the entire range of firing rates common to both conditions, at any given mean count (Fig. $4 A$, shaded area), variability was greater during undirected song than during directed song. To quantify this difference across the population of LMAN neurons, for each neuron we averaged the Fano factor for all $100 \mathrm{~ms}$ windows that had a mean spike count between one and two spikes (firing rate $=10-20 \mathrm{~Hz}$ ) (Fig. $4 A$, shaded area). Given the marked differences in firing rates across conditions, this allowed comparison of spike count variability for the maximum number of neurons. Even when the firing rate was the same, variability was significantly greater during undirected song than during directed song (Fig. $4 B$ ). Thus, firing rate alone could not account for the context-dependent differences in spike count variability.

\section{Spike timing precision is high during directed singing}

We have already shown that the firing pattern of individual LMAN neurons is significantly more reproducible (Fig. 3A,B) and the spike count is significantly more reliable (Fig. $3 C-E$ ) across repeated trials of directed song compared with undirected song. To directly measure the precision of spike timing across trials, we examined the variability in the times of the first spike during "reliable firing events" (see Materials and Methods) (Fig. $5 A-C$ ) (Mainen and Sejnowski, 1995; Berry et al., 1997). Our goal was to measure the precision of the most salient events related to the song. For each neuron, we first identified periods of elevated firing, or spike "events," during the motif. To exclude cases that might have been caused by factors unrelated to the song (cf. Reinagel and Reid, 2002; Khumbani et al., 2007), we only analyzed those events in which at least $50 \%$ of the trials contained a spike. For each event, we accumulated the time of the first spike across all trials and computed the SD of these spike times (Fig. $5 D$ ). We averaged these values across all events during the motif to obtain a measure of temporal jitter for each neuron. This mea-

$\leftarrow$

in LMAN activity increased significantly during DIR compared with when the bird was silent $(p<0.0001$; paired sign test). In contrast, spike count reliability was significantly lower during UNDIR than during nonsinging periods ( $p<0.0001$; paired sign test). Across the entire population of LMAN neurons, spike count variability was greater during UNDIR than during DIR (for neurons with recordings in both social conditions (filled symbols connected by lines), $p<$ 0.0001 , paired sign test; for neurons recorded in only one condition (open symbols), $p=0.003$, $\mathrm{M}-\mathrm{W}$ test). 
A
LMAN $_{1}$

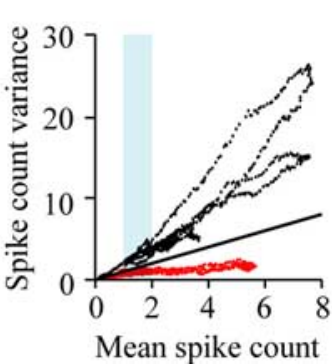

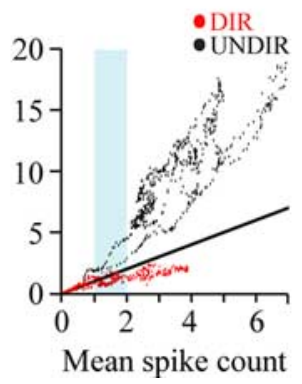

B

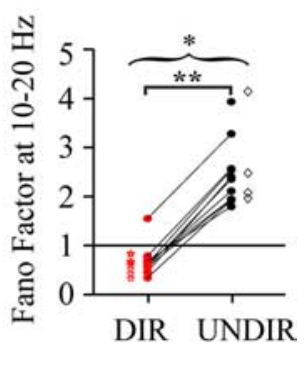

Figure 4. Firing rate alone cannot account for context-dependent differences in spike count variability. $\boldsymbol{A}$, Mean spike count versus variance in $100 \mathrm{~ms}$ sliding windows for the two cells shown in Figure 3 during DIR (red) and UNDIR (black). Even when the firing rate is the same, spike count variability is greater during UNDIR. Diagonal line corresponds to $F=1$. Shaded region highlights the range of spike count variance in windows with $1-2$ spikes. $\boldsymbol{B}$, The mean $F$ for all 100 ms windows that had between 1 and 2 spikes is plotted for individual neurons. Variability is greater during UNDIR compared with during DIR, even when firing rate is matched (for neurons recorded in both conditions, $p=0.002$, paired sign test; for neurons recorded in one condition, $p=$ $0.006, M-W$ test)

sure represents an upper estimate of the spike timing precision of LMAN neurons during singing.

During directed singing, spike timing precision could be quite high. For the two neurons in Figure 5, the temporal jitter was 1.8 $\mathrm{ms}$ and $2.5 \mathrm{~ms}$, respectively. Across the population of LMAN neurons $(n=23)$, reliable spike events were identified in all but one neuron, and the mean temporal jitter was $2.5 \pm 0.5 \mathrm{~ms}$ (range: $1.8-3.4 \mathrm{~ms}$ ), similar to but more precise on average than the result reported by Leonardo (2004) using a different measure. The high degree of temporal precision possible during directed singing suggests that the timing of firing events is one important aspect of signaling for these sensorimotor neurons.

Spike timing precision was lower during undirected singing than during directed singing. Across the population of LMAN neurons recorded during undirected singing, reliable spike events were identified in 9 of 19 neurons, consistent with the greater variability in firing pattern across repeated undirected trials (Fig. $3)$. For neurons that did produce reliable spike events during undirected song, the mean temporal jitter was $2.9 \pm 1.8 \mathrm{~ms}$ (range: $1.3-7.11 \mathrm{~ms}$ ), indicating that LMAN neurons can generate precisely timed events, even in the undirected condition.

\section{Bursts contribute to context-dependent differences in trial-by-trial variability}

In other systems, bursting can be associated with high trial-bytrial variability in neural activity (Kara et al., 2000). Because our study revealed that LMAN neurons fire bursts of spikes predominantly during undirected song (Fig. $2 \mathrm{~B}$ ), we examined the contribution of bursts to the context-dependent difference in spike count variability. To do so, we treated bursts as unitary events and retained only the first spike of each burst along with the "isolated" spike events, which occur outside of the context of bursts. Figure 6 illustrates this analysis for the neuron shown in Figure 1. When bursts were treated as unitary events, context-dependent differences in firing rate and spike count variability were substantially reduced (Fig. 6A-F). When we calculated the Fano factor in a 30 ms sliding window, during undirected song, many more windows had variance close to the mean count (Fig. 6C) (timeaveraged $F=1.10$ ) than in the original data (time-averaged $F=$ 1.82) (Fig. 3D, left). In contrast, spike count variability during directed song did not change substantially (time-averaged $F$ without bursts $=0.66$; time-averaged $F$ with bursts $=0.65)$. Across all LMAN neurons, the time-averaged $F$ was significantly lower in the absence of bursts compared with the original data in the undirected condition (means: 1.03 versus 2.00, respectively; $p<0.001$, paired sign test) (compare Figs. $6 D$ and $3 E$ ). This was also true in the directed condition, although much less so (means: 0.67 vs $0.70 ; p<$ 0.0001 , paired sign test). Similar results were obtained when we calculated $F$ with a $100 \mathrm{~ms}$ sliding window (undirected means: 1.17 vs $2.40 ; p<0.0001$, paired sign test; directed means: 0.57 versus 0.62 ; $p=0.0001$, paired sign test). Thus, bursting contributes significantly to spike count variability.

Treating bursts as unitary events, however, did not completely abolish the context-dependent differences in spike count variability (Fig. $6 C, E$ ). In the absence of bursts, spike count variability remained significantly higher during undirected song compared with during directed song (Fig. 6D) (mean time-averaged $F$ in 30 ms windows: 1.03 vs $0.66 ; p=0.0001$, paired sign test), even when firing rate was taken into account (Fig. $6 F$ ) (mean $F$ at $10-20 \mathrm{~Hz}$ : 1.32 vs $0.64 ; p=$ 0.001 , paired sign test). Similar results were obtained when we analyzed only the isolated spike events (mean time-averaged $F$ in 30 ms windows: 1.04 vs $0.70 ; p<0.001$ ), indicating that isolated spikes also contribute to context-dependent differences in spike count variability.

Bursts could contribute to higher neural variability during undirected song in at least two ways: 1 ) if the number of spikes within a burst was variable; and 2) if bursts occurred at different times in the motif across trials. With respect to the first possibility, we found that the number of spikes per burst varied during undirected song, both within a trial and across trials. In contrast, during directed song, if the neuron did fire a burst, the number of spikes per burst was typically two. For example, for the LMAN neuron shown in Figure 1, the mean number of spikes per burst was $3.0 \pm 1.5$ during undirected song (range: $2-13$ ) and $2.0 \pm 0.0$ during directed song. Across all neurons, the mean number of spikes per burst was significantly greater during undirected song than during directed song (Fig. 7A) (mean \pm SD: $3.10 \pm 0.3$ versus $2.1 \pm 0.1$ ). Moreover, variability in the number of spikes per burst was also significantly greater during undirected song than during directed song (Fig. $7 B$ ) [mean coefficient of variation (c.v.): 0.43 versus 0.08 ].

Next, to investigate whether variable timing of burst occurrences across trials also contributes to greater variability in LMAN activity during undirected song, for each neuron we computed the correlation coefficients between all pairs of undirected trials using only the burst onsets (Fig. $8 A, B$, compare the rasters), and then averaged across all pairs of undirected trials. Across the population of LMAN neurons, the mean correlation in burst onsets across repeated renditions of the motif was $0.10 \pm 0.02$ (SEM), indicating a high degree of variability in the firing pattern of burst onsets. Thus, greater variability in LMAN activity during undirected song derives from variation not only in the structure of bursts, but also in the timing of their onsets.

Bursts, however, were not the sole source of variability during undirected singing. Isolated spikes, which occur outside of the 

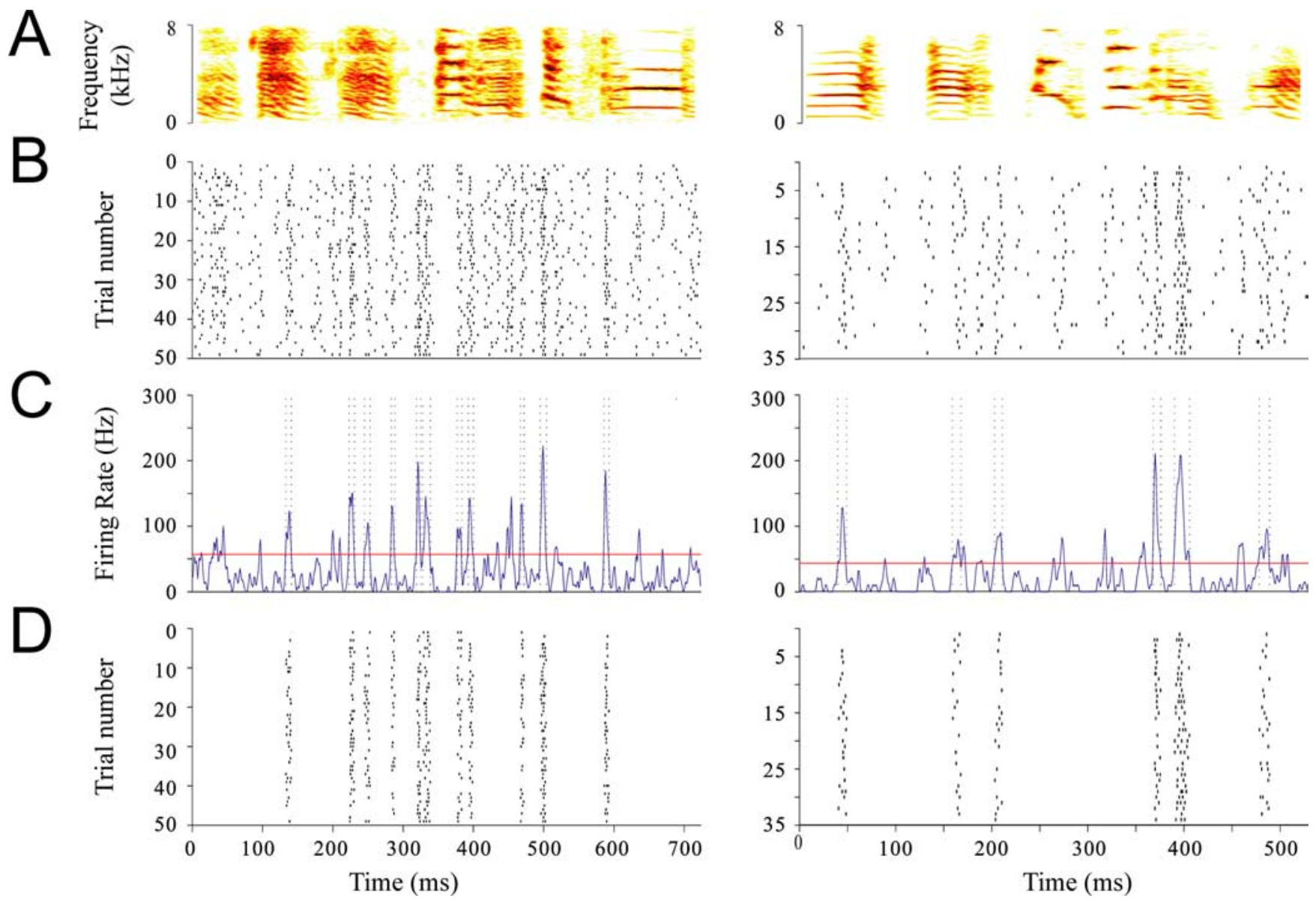

Figure 5. Spike timing precision of LMAN neurons is high during DIR. A, Spectrograms of the motifs of two different birds (b39b14 and p49w84). B, Raster plots of the singing-related activity of two single LMAN neurons during multiple renditions of DIR. C, Histograms of the time-warped spikes smoothed with a Gaussian filter ( $S D=4 \mathrm{~ms}$ ). A threshold (horizontal line, set at the mean firing rate +4 SD) was used to identify reproducible spike events (indicated by dotted lines). Events in which the cell failed to spike in at least $50 \%$ of the trials were omitted from further analysis. $\boldsymbol{D}$, Raster plot of the reliable spike events. Spike timing precision, or "temporal jitter," was defined as the SD of the first spike in the event, averaged over all reliable events during the motif. For the two examples shown, the temporal jitter was $1.8 \mathrm{~ms}$ and $2.5 \mathrm{~ms}$, respectively. Spike timing precision was similar regardless of the width of filter used (see Materials and Methods; range of temporal jitter for each neuron across the different filter widths $=1.8-2.0$ and $2.2-2.7 \mathrm{~ms}$ ) as well as when the spikes were binned without smoothing (temporal jitter $=1.8 \mathrm{~ms}$ and $1.9 \mathrm{~ms}$, respectively).

context of bursts, were also highly variable in their timing across repeated undirected trials. Across the population of LMAN neurons, correlations in the timing of isolated spikes across repeated motif renditions were also low [mean pairwise correlation coefficient $=$ $0.11 \pm 0.01(\mathrm{SEM})]$. Thus, variability in spike timing in general (i.e., for both burst onsets and for isolated spikes) contributes to greater variability of LMAN activity during undirected song.

\section{Similarity of average firing patterns across social context}

Despite greater cross-trial variability in the firing pattern during undirected singing than during directed singing, the average pattern of activity across many trials was remarkably similar across behavioral conditions (Figs. $1 F, 3 C$ ). To quantify the degree of similarity, we calculated the correlation coefficient between the mean firing patterns for each LMAN neuron in the two behavioral conditions. For the neuron in Figure 1, the correlation coefficient was 0.84 . Across all neurons with paired recordings, the mean correlation coefficient was $0.69 \pm 0.13$ (range: $0.41-0.87$ ). Thus, each LMAN neuron generated a characteristic song-locked average firing pattern, regardless of social context and the attendant differences in variability.

Our finding that the average pattern of activity was similar across behavioral conditions despite significantly more variable burst firing during undirected singing raises the question of whether the timing of bursts was completely random with respect to the song. If so, bursts could introduce noise without obscuring the average pattern of singing-related activity. Alternatively, despite some variability in their precise timing, burst onsets could, on average, exhibit a pattern across repeated trials that is similar to the activity during directed song. To examine these possibilities, we compared the motif-aligned raster of burst onsets with that of the complete spike train for each LMAN neuron during undirected singing (Fig. $8 A, B$ ). We found that the characteristic song-locked firing pattern of each LMAN neuron was apparent in the timing of burst onsets across many trials. Indeed, the correlation coefficient between the mean firing pattern of burst onsets and that of the complete spike train was high (see Materials and Methods). For the representative LMAN neuron in Figure 8, the correlation coefficient was 0.76 , and across the population of LMAN neurons, the correlation coefficient was $0.73 \pm 0.07$ (mean $\pm \mathrm{SD}$ ), indicating that the probability of bursting was not independent of the overall firing pattern during undirected singing.

\section{Firing pattern correlations across populations of LMAN neurons}

Previous studies of singing-related activity in LMAN found that the average pattern of multiunit activity in LMAN was similar 


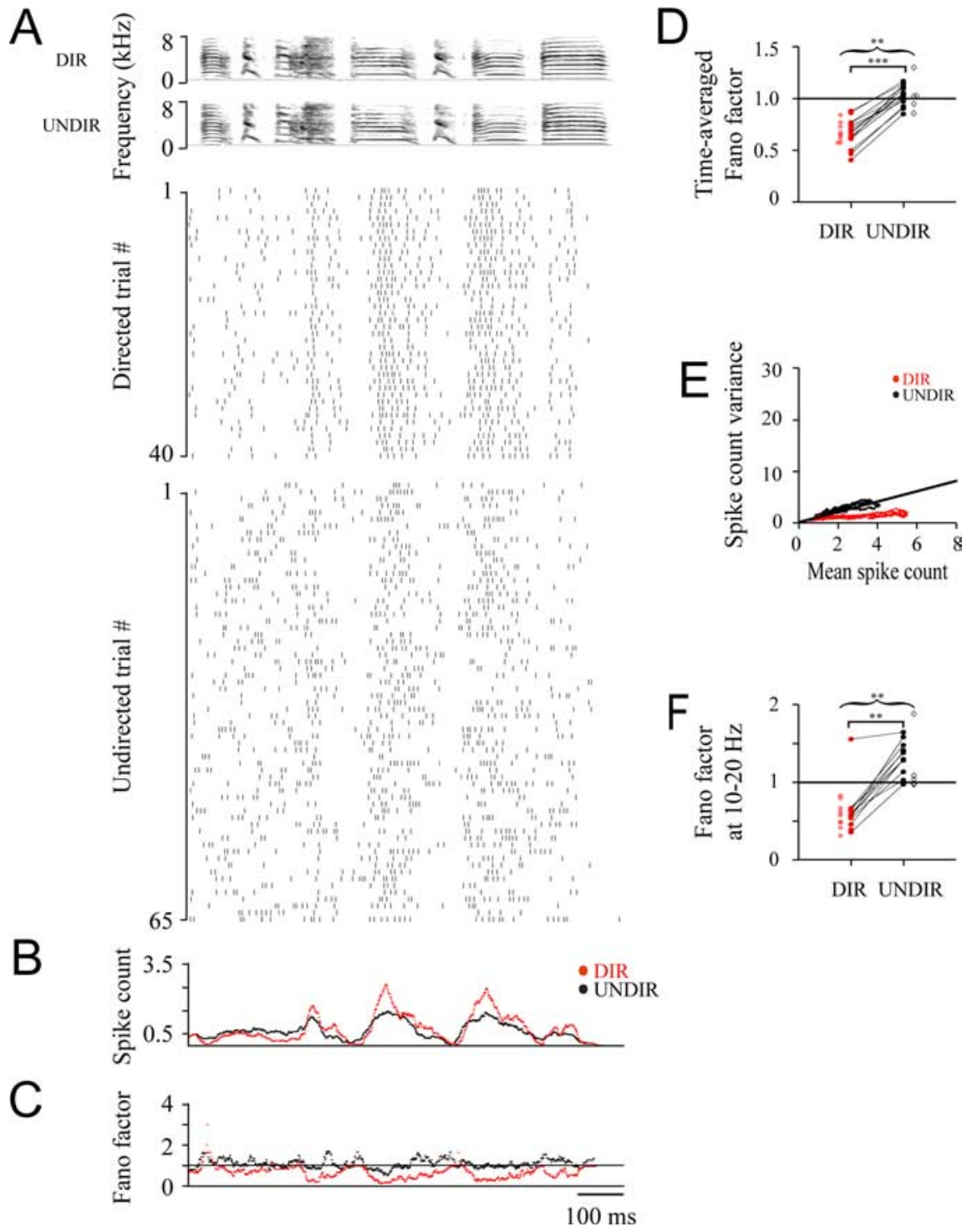

Figure 6. Context-dependent differences in bursting contribute to greater spike count variability during UNDIR. $A$, Singingrelated activity when bursts are treated as unitary events and only the first spike per burst is retained along with the isolated spike events. Data are from the cell shown in Figure 1 (same as $L_{M A N}$ in Figs. 3 and 4). Compared with the original data (Fig. 1E,F), the firing rate is substantially reduced during UNDIR, and there is no longer a difference in firing rate across social context. The pattern of activity, however, is still more reproducible during DIR (top) than during UNDIR (bottom). $\boldsymbol{B}$, Mean spike count (firing rate) in a 30 ms sliding window when isolated spikes and the first spike per burst are retained. $C$, Fano factor $(F)$ through the course of the motif for the same data. Context-dependent differences in spike count variability are still present but reduced compared with the original data (Fig. 3D, left). D, The time-averaged $F(T=30 \mathrm{~ms})$ is plotted when bursts are treated as unitary events. Although $F$ is substantially lower in the absence of bursts (compare with Fig. $3 E$ ), spike count variability is still greater during UNDIR than during DIR (for neurons with recordings in both conditions (filled symbols connected by lines, $p=0.0001$, paired sign test; for neurons with recordings in only one behavioral condition, $p=0.003, \mathrm{M}-\mathrm{W}$ test). Some symbols have been offset horizontally to reduce overlap. $\boldsymbol{E}$, Variance versus mean spike count in $100 \mathrm{~ms}$ windows for the same cell when bursts are treated as unitary events. At the same firing rate, variability is greater during UNDIR. Compared with the original data (Fig. $4 A$, left), however, many points lie along the line $F=1$ during UNDIR. $F$, Mean $F$ across all 100 ms windows that had between 1 and 2 spikes is plotted for individual neurons when bursts are treated as unitary events. At the same firing rate, variability is reduced compared with the original data (Fig. $4 B$ ) but remains significantly greater during UNDIR than during DIR (for neurons recorded in both conditions, $p=0.0001$, paired sign test; for neurons with recordings in only one behavioral condition, $p=0.003, \mathrm{M}-\mathrm{W}$ test).

trains of all LMAN neurons recorded in the same bird to a common reference motif revealed that different LMAN neurons exhibit distinct time-varying firing patterns during song $(n=8,5,3,3$, and 2 neurons in 5 birds). Figure 9 shows an example of the singing-related activity of eight LMAN neurons from one bird (b39b14) during repeated trials of directed song (syllables abcdef; mean number of trials per neuron $=34 \pm 14(\mathrm{SD})$; range: 12-55). Recordings of the different neurons were performed at intervals of $1 \mathrm{~h}$ to several days. Across the population, reliable differences in spike timing are apparent. For example, one neuron (green; third from the top) fired consistently before the onset of syllable d, while another neuron (red; sixth from the top) was silent during the interval between syllables $c$ and $d$. In addition, these two neurons fired at consistently different times during syllable e. The mean correlation coefficient of the firing patterns of all pairs of LMAN neurons recorded in this bird was $0.20 \pm 0.17$ (SD). Across all birds, the mean correlation coefficient between different LMAN neurons was $0.19 \pm 0.19$ during directed singing (45 pairwise correlations; range: -0.33 to 0.62 ). Neurons often fired out of phase with one another, and only one of 45 pairwise correlations was $>0.50$. Our finding that different LMAN neurons in the same bird consistently fire spikes at different times during the song contrasts sharply with the findings of previous multiunit recordings. Rather than sending a uniform signal to the motor pathway, subsets of LMAN neurons may jointly encode particular features of song.

\section{Social context-dependent differences in LMAN activity preceding song initiation and following song termination}

In addition to increased LMAN activity during undirected singing, Hessler and Doupe (1999b) reported that the level of LMAN activity was consistently altered before and after bouts of undirected song. Along with a sharp rise in activity $\sim 30-90$ ms before song initiation, a more gradual rise in activity level preceded song initiation in two-thirds of their recording sites. In contrast, LMAN activity was consistently suppressed following termination of undirected song.

across multiple recording sites in the same bird, raising the possibility that the entire nucleus sends a uniform singing-related signal to the motor pathway (Hessler and Doupe, 1999b). To directly examine this hypothesis, we compared the singingrelated activity of multiple single neurons in the same bird. We focused on directed song activity because trial-by-trial variability is lower than during undirected song. Alignment of the spike
To directly investigate presinging activity of single LMAN neurons, we analyzed neural activity leading up to song for cases in which the bird was silent for at least $2 \mathrm{~s}$ before song initiation. Figure 10, $A$ and $B$, shows the activity of two representative LMAN neurons during an epoch from 1500 ms before song initiation to $200 \mathrm{~ms}$ after song initiation. Undirected spike trains are plotted below directed spike trains. When males were alone, sin- 

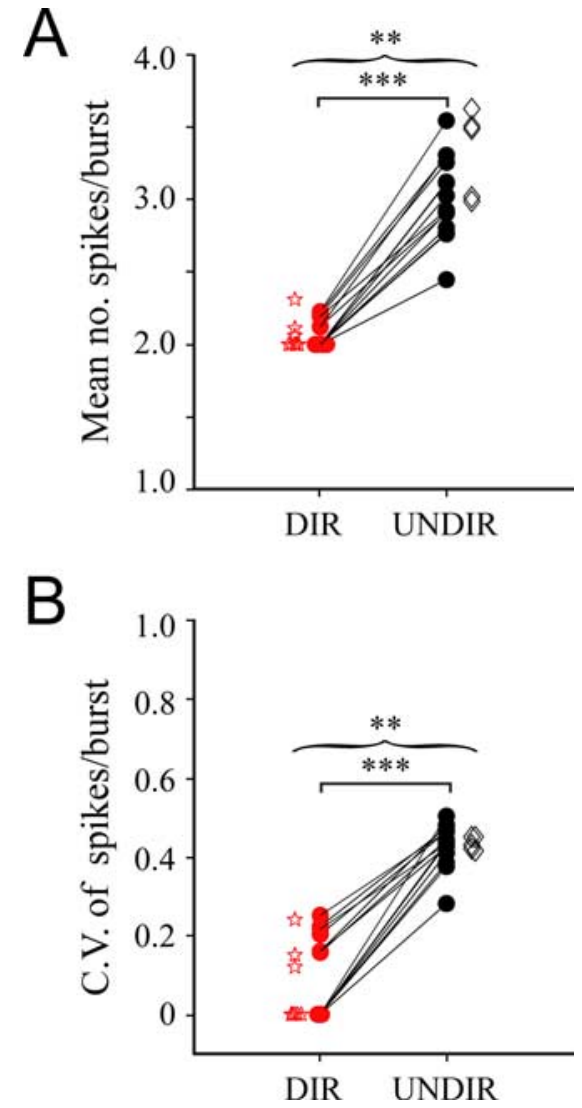

Figure 7. Variability in the number of spikes per burst is greater during UNDIR. Across the population of LMAN neurons, both the mean number of spikes per burst $(\boldsymbol{A})$ and the variability in the number of spikes per burst (B), expressed as the coefficient of variation (c.v.), are significantly greater during UNDIR than during DIR (some symbols have been offset horizontally to reduce overlap). Context-dependent differences in the structure of bursts were apparent across the population and in every neuron with recordings in both behavioral conditions (filled symbols; lines connect data from the same neuron; $p=0.0001$, paired sign test).

gle LMAN neurons gradually increased their firing rates hundreds of milliseconds before song onset, consistent with the findings of Hessler and Doupe (1999b). For 18 of 19 neurons, firing rate in a $500 \mathrm{~ms}$ interval from 600 to $100 \mathrm{~ms}$ before undirected song was greater than the average spontaneous firing rate (Fig. $10 C, D)(p<0.0001$, paired sign test). In contrast to the presong ramp-up of LMAN activity in the undirected condition, the firing rate of single LMAN neurons did not consistently increase before initiation of female-directed songs (Fig. 10A-D). Across the population of LMAN neurons, firing rate in the epoch from 600 to $100 \mathrm{~ms}$ before initiation of directed song was not significantly different from the spontaneous firing rate (Fig. 10C,D).

Context-dependent differences in LMAN activity were also present following song termination. Consistent with Hessler and Doupe (1999b), we found that the firing rates of single LMAN neurons were suppressed following song termination when the male was alone (Fig. $11 A-C$ ). To quantify the maximal inhibition of LMAN activity following song, we computed the firing rate in a $500 \mathrm{~ms}$ interval from 100 to $600 \mathrm{~ms}$ after the last song syllable. In the undirected condition, activity during this epoch was lower than the average level of spontaneous activity in 16 of 19 neurons (Fig. $11 D)(p<0.005$; paired sign test). In contrast, LMAN activity in the directed condition was not consistently suppressed following song termination (Fig. 11A-D). While contextdependent differences in postsong activity may stem mechanisti- cally from the marked differences in burst firing across conditions, they could have significant consequences for song production and plasticity. For example, following termination of undirected song bouts, suppression of LMAN activity might provide a window during which intracellular signaling mechanisms in RA, triggered by burst firing in LMAN, could act to modify synapses.

\section{Discussion}

By recording many trials of single unit activity during behavior, we have shown that individual LMAN neurons rapidly switch between two distinct firing modes, characterized by a dramatic change in burst firing, depending on social context. When male zebra finches sing to females, LMAN neurons exhibit reliable firing of single spikes across repeated trials. In contrast, during undirected singing, the same LMAN neurons exhibit strikingly variable burst firing. Although previous IEG and multiunit recording studies demonstrated that the activity of the LMAN population changes with social context, our single unit recordings reveal the nature of the changes. In addition, by recording from multiple single neurons in the same bird, we found that individual LMAN neurons send distinct patterned signals to the motor pathway. These results challenge previous notions that LMAN sends a uniform signal or random "noise" to its targets (Doupe, 1997; Hessler and Doupe, 1999b; Fiete et al., 2007). Together, our findings suggest that individual LMAN neurons not only introduce variability into song, which could enable motor exploration, but also code song features, perhaps biasing song toward a target. Moreover, the degree of variability around the underlying firing pattern of each neuron is markedly regulated by social context, which changes the probability of bursting.

\section{Context-dependent bursting}

Our single unit recordings showed that behavioral context strongly modulates the probability of burst firing of LMAN neurons. Across all neurons, more than half of all spikes fired during undirected motifs occurred in bursts compared with $<10 \%$ of spikes during directed motifs.

Such context-dependent differences in burst probability could explain the finding that egr-1 is induced in more AFP neurons when males sing alone than when they sing to females (Jarvis et al., 1998). Activation of IEGs is highly sensitive to the pattern of stimulation (Sheng et al., 1993; Worley et al., 1993), and bursts can be more effective at inducing IEGs than tonic activity, even when the overall number of spikes is identical. During undirected singing, more LMAN neurons may appear to be active because marked bursting drives greater IEG induction. Our recordings of single units, however, show that individual LMAN neurons exhibit patterned activity during both directed and undirected singing.

Context-dependent bursting in LMAN could contribute to song variability and plasticity through multiple mechanisms. First, burst firing may be more effective than single spikes at driving postsynaptic responses (Lisman, 1997). In this manner, bursting in LMAN could acutely increase the signal-to-noise ratio of neuronal responses in RA. Second, LMAN bursting could enhance plasticity in RA, perhaps via enhanced calcium influx through NMDA receptors on RA neurons that also receive premotor inputs from HVC (Mooney and Konishi, 1991; Stark and Perkel, 1999). Finally, bursting in LMAN could contribute to song plasticity by regulating the release of trophic factors (Balkowiec and Katz, 2000), which can influence the structural 
A

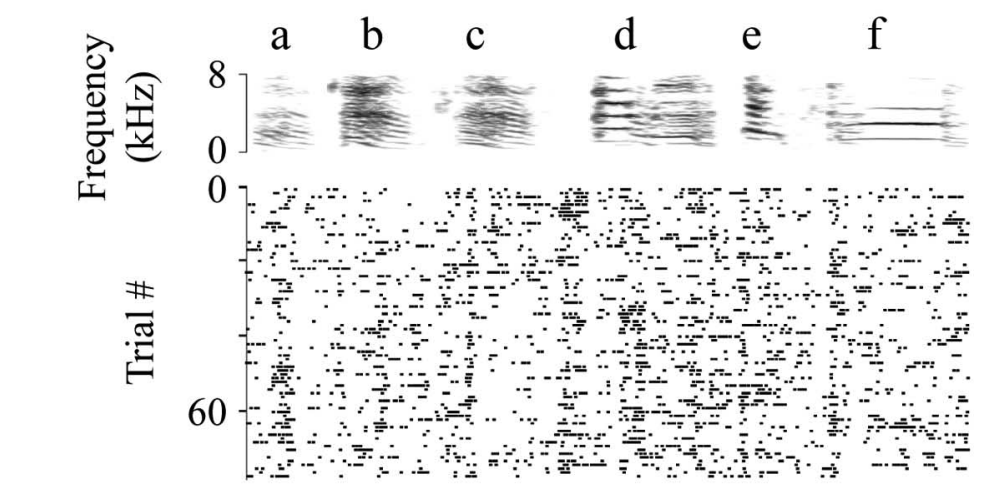

B

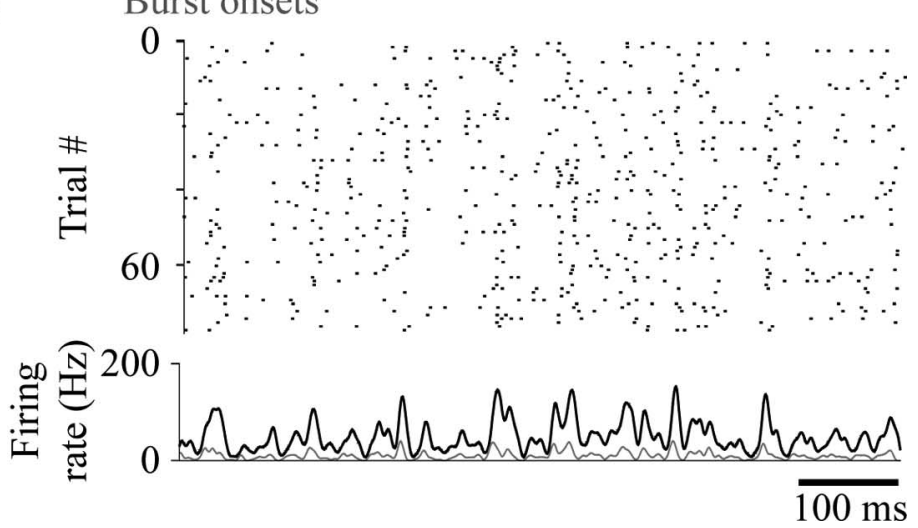

Figure 8. Precise timing of burst onsets is low during UNDIR, although not independent of the overall firing pattern. $A$, Raster plot of the motif-aligned activity of one LMAN neuron during UNDIR (b39b14.11). (For comparison with the directed activity, see Fig. 9, fifth unit from the top). $\boldsymbol{B}$, Top, Raster plot of onsets of bursts (instantaneous firing rate $\geq 200 \mathrm{~Hz}$ ) for the same neuron. The timing of burst onsets was highly variable across UNDIR trials (mean pairwise correlation coefficient $=0.09$ ). Bottom, The mean firing pattern of the complete spike train (black) is overlaid with the mean firing pattern of the burst onsets (gray). Despite cross-trial variability in burst onsets, the average firing pattern of burst onsets was similar to that of the complete spike train during UNDIR (correlation coefficients between the mean firing patterns $=0.76$ ).

organization of the motor pathway (Johnson et al., 1997; Kittelberger and Mooney, 1999, 2005).

\section{Trial-by-trial variability and motor learning}

Prior modeling has hypothesized that song learning occurs through a process of trial-and-error in which variable songs are produced ("motor exploration") and auditory feedback-based evaluation mechanisms selectively reinforce motor patterns that produce better versions (Sutton and Barto, 1998; Doya and Sejnowski, 2000; Fiete et al., 2007). Even after song is well learned, however, trial-by-trial variability in song persists. Such variability may be important for continued motor exploration to maintain or optimize behavior, especially in response to age- or injuryrelated changes in motor areas. Indeed, adult songbirds can use such residual variability to adaptively modify their song (Tumer and Brainard, 2007).

Several lines of evidence suggest that LMAN activity generates the variability in song output that is critical for trial-and-error learning throughout life: (1) social factors that modulate variability in AFP activity also modulate variability in song (Sossinka and Böhner, 1980; Hessler and Doupe, 1999a; Kao et al., 2005; Olveczky et al., 2005); (2) lesions or inactivation of LMAN sharply reduce song variability and plasticity (Bottjer et al., 1984; Scharff and Nottebohm, 1991; Williams and Mehta, 1999; Brainard and
Doupe, 2000; Olveczky et al., 2005; Kao and Brainard, 2006); and 3) artificial manipulation of LMAN activity can introduce variability into song (Kao et al., 2005). In this study, we have shown that variability in firing patterns of individual LMAN neurons is tightly regulated, consistent with the idea that LMAN activity dynamically modulates moment-bymoment variability in song and may contribute to the rapid switching of motor activity between variable and precise states.

This study of LMAN single unit activity advances our understanding of song learning in several ways. First, models of trialand-error learning predict that the amount of variability in motor output should depend on the current state of performance, decreasing when song is close to the target. Indeed, we found that trial-bytrial reliability in LMAN activity in adult birds is greater than that of juvenile birds engaged in sensorimotor learning: mean correlation between all pairs of trials during undirected song was $0.24 \pm 0.08$ (SD) for adults in this study versus $0.054 \pm 0.34$ for juveniles in the study of Olveczky et al. (2005). Similar increases in the reliability of task-related neural activity have been correlated with learning and improved motor performance in other systems, including mammalian striatum (Kargo and Nitz, 2004; Barnes et al., 2005).

Second, models of trial-and-error learning often assume that the neural variability enabling motor exploration is random (Sutton and Barto, 1998; Fiete et al., 2007). Our data, however, show that not only does undirected activity exhibit the same singing-related pattern, on average, as directed activity, but so too do the bursts that are the primary source of variability. Thus, in adult birds at least, bursting is not simply random and may contain signals that could "bias" motor output (Doya and Sejnowski, 1998; Troyer and Doupe, 2000; Kao et al., 2005). For example, patterned activity in LMAN may guide vocal output toward the target by specifically injecting variability during some syllables but not others. Similar biasing signals have been postulated to be an important function of cortical and striatal activity in other systems (Miller, 2000; Hikosaka et al., 2002).

\section{Temporal precision of LMAN activity}

We found that LMAN neurons are capable of reliably firing at the same location in song across repeated trials with a high degree of temporal precision, especially during directed singing. While many spike events exhibited variability in their timing across trials, jitter in the timing of the most reliable firing events was as low as a few milliseconds, similar to that observed in many systems (Mainen and Sejnowski, 1995; Berry et al., 1997; Reinagel and Reid, 2002; Uzzell and Chichilnisky, 2004). Precise timing of LMAN activity during singing may be important for driving adaptive changes in the motor pathway. RA receives precisely timed afferent input from the premotor nucleus HVC during singing (Fig. 1A) (Hahnloser et al., 2002). Given our finding that 

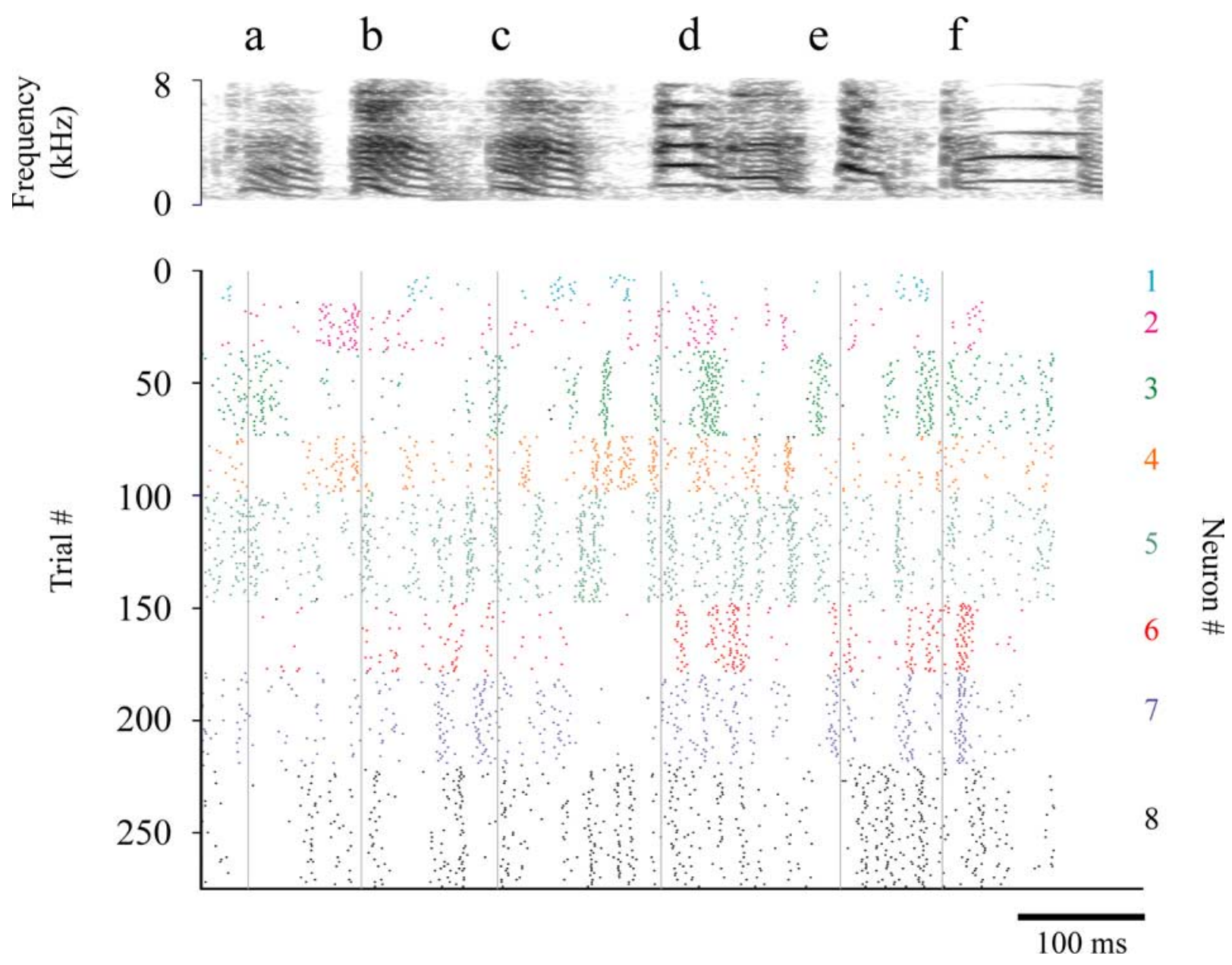

Figure 9. Singing-related activity of a population of LMAN neurons in an adult zebra finch. Raster plot of the song-aligned activity of eight LMAN neurons recorded in one bird (b39b14). Each row represents the singing-related activity during one rendition of the motif. Different colors indicate different LMAN neurons. Recordings were made sequentially over a period of hours to days, and the activity has been aligned to a common time axis using the reference motif shown at the top (see Materials and Methods). Vertical lines indicate syllable onsets. Songs and neural activity were collected when the male sang to a female (directed). Individual LMAN neurons generated precise, distinct patterns of activity during the motif.

LMAN neurons can also generate precise song-locked firing patterns, the relative timing of separate inputs from HVC and LMAN may be important for inducing functional changes in RA. For example, coincident input from the two nuclei may facilitate strengthening of RA synapses.

Although spike timing precision of LMAN neurons could be quite high during directed singing, it is still an order of magnitude lower than that observed in RA (Chi and Margoliash, 2001), perhaps reflecting differences in contributions of the two nuclei to song production and plasticity. While RA is required to generate motor commands that precisely coordinate vocal and respiratory musculature for song, LMAN's primary function may be to generate song variability and plasticity.

\section{Ensemble coding of song in LMAN}

In contrast to the notion that all LMAN neurons in a given bird send a uniform signal to RA (Hessler and Doupe, 1999b; Fiete et al., 2007), we found that different LMAN neurons in the same bird exhibit distinct time-varying firing patterns during directed song. Several factors can account for the different findings. First, Hessler and Doupe (1999b) analyzed a smoothed representation of the activity of multiple LMAN neurons. In addition, they did not compensate, as we did here, for natural differences in syllable and interval durations, resulting in variable alignment of neural activity across trials, which could obscure differences in firing patterns across sites (Chi and Margoliash, 2001). Finally, by using two electrodes spaced $\sim 300-600 \mu \mathrm{m}$ apart (versus a single electrode used by Hessler and Doupe), our recordings may have sampled a greater diversity of sites, including different anatomic compartments in LMAN.

The diversity of temporally precise firing patterns across the nucleus, similar to that observed in RA (Leonardo and Fee, 2005), suggests that subsets of LMAN neurons jointly encode particular features of song. Consistent with the notion of ensemble coding, we have shown previously that focal stimulation in LMAN can elicit specific changes in song (Kao et al., 2005). For example, a fixed pattern of stimulation at one site in LMAN may cause a change in the frequency of one syllable but not another. Similarly, different effects can be elicited in a particular syllable when the site of stimulation is explicitly varied, suggesting that local ensembles code specific song features. Together with our new finding that different LMAN neurons predictably fire at different times in song, these results suggest that LMAN is functionally compartmentalized, perhaps reflecting topography within the AFP and in the projections from LMAN to RA (Johnson et al., 1995; Luo et al., 2001). 
A

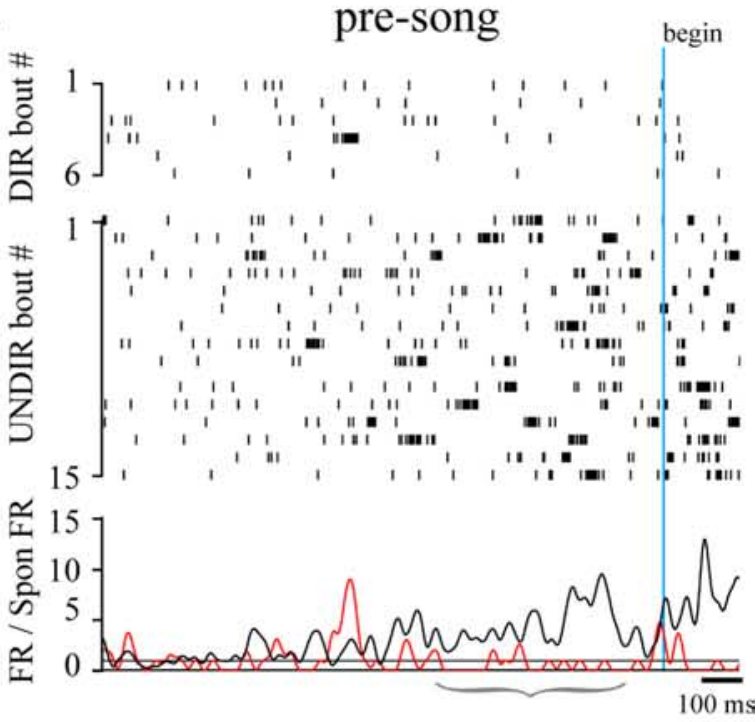

$\mathrm{B}$
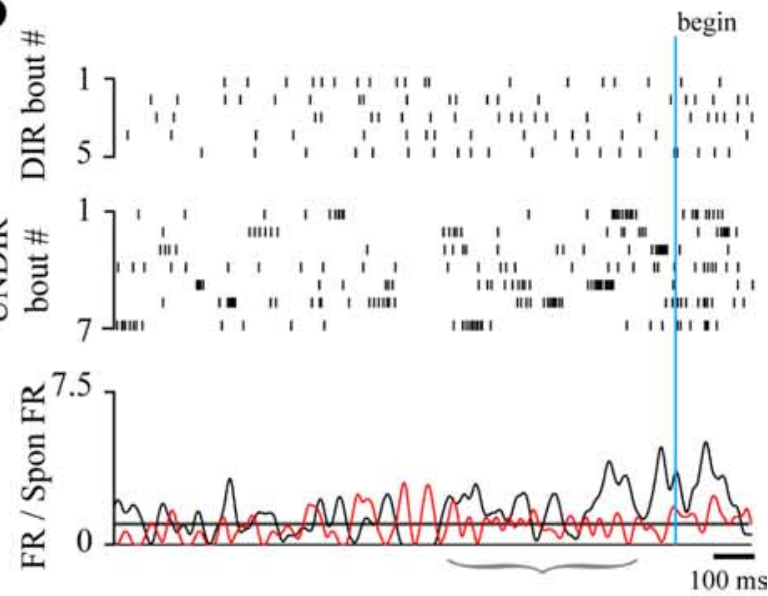

C

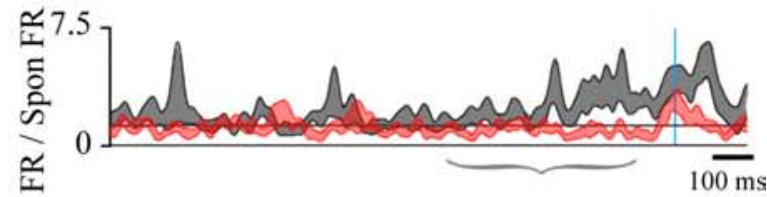

$\mathrm{D}$

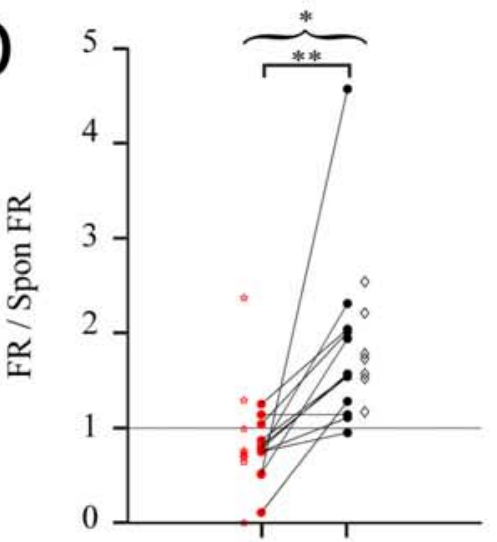

DIR UNDIR

Figure 10. Firing rate of LMAN neurons gradually increases before song initiation in the UNDIR condition but not in the DIR condition. $A, B$, Raster plot of the activity of two representative LMAN neurons during an epoch from $1500 \mathrm{~ms}$ before song initiation to $200 \mathrm{~ms}$ after song
Externally triggered versus internally generated singing

Prior work has shown that some HVC neurons exhibit a gradual increase in firing rate as much as several seconds before song onset, perhaps reflecting a role in motor preparation or initiation (McCasland and Konishi, 1987; Dave et al., 1998). Here, we found that single neurons in LMAN also ramp up their activity several hundreds of milliseconds before the onset of undirected song but not before female-directed song. Such contextdependent differences in presong LMAN activity are highly reminiscent of observations in mammalian striatum that activity gradually builds up before movements when they are internally generated but not when they are triggered by external sensory cues (Schultz and Romo, 1992; Lee and Assad, 2003). Thus, for both birds and mammals, behavioral context can markedly influence initiation of motor behavior. Without external cues, initiation of self-timed behaviors (e.g., undirected singing), may occur only after activity in cortical-basal ganglia circuits increases until it exceeds a threshold level (Lee and Assad, 2003). External cues (here, a female conspecific), however, may rapidly drive activity above threshold, perhaps via neuromodulators. Indeed, sensory cues can stimulate motor behaviors in parkinsonian patients who have difficulty initiating the same movements when they are internally motivated (Glickstein and Stein, 1991). Alternatively, external cues may act by lowering the neural threshold for motor behaviors. Consistent with this possibility, LMAN activity both before and during singing is lower in the directed compared with the undirected condition.

\section{Potential sources of state-dependent modulation of LMAN activity}

In many systems, the firing mode of burst-capable neurons is gated by modulatory input (for review, see Krahe and Gabbiani, 2004). Neuromodulators can modify firing rate, spike timing precision (Billimoria et al., 2006), and burst probability (for review, see Marder and Bucher, 2007). In songbirds, contextdependent differences in AFP activity may derive from socially driven alterations in neuromodulatory inputs. For example, experimentally induced reduction of systemic norepinephrine alters social context-dependent expression of egr-1 in Area X (Castelino and Ball, 2005). State-dependent dopamine levels may also mediate differences in AFP firing. The ventral tegmental area (VTA) sends a dense dopaminergic projection to the AFP (Lewis et al., 1981; Bottjer et al., 1989), and dopamine levels in Area X are higher during directed singing than during undirected singing (Sasaki et al., 2006). Moreover, dopamine can directly modulate synaptic inputs and intrinsic excitability of AFP neurons (Ding and Perkel, 2002; Ding et al., 2003).

Alternatively, or in addition, context-dependent differences 

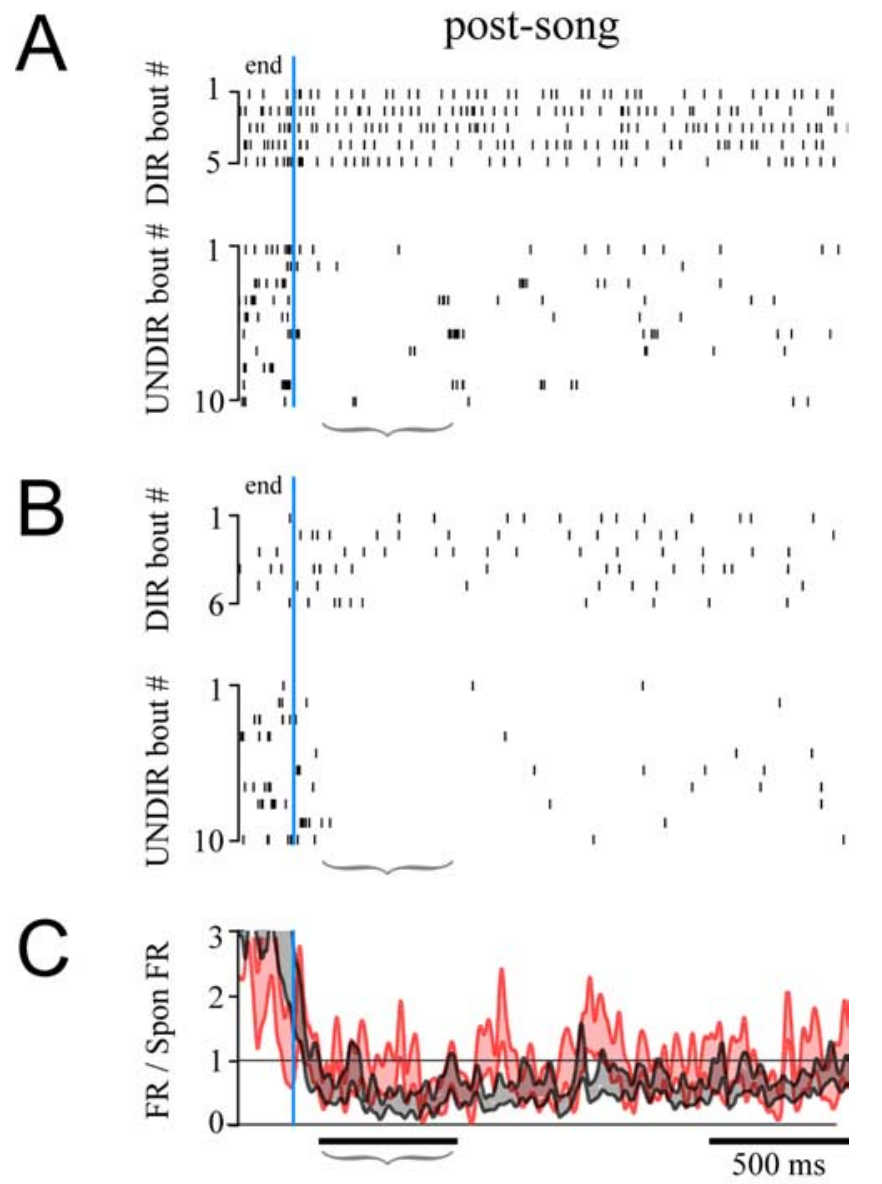

D

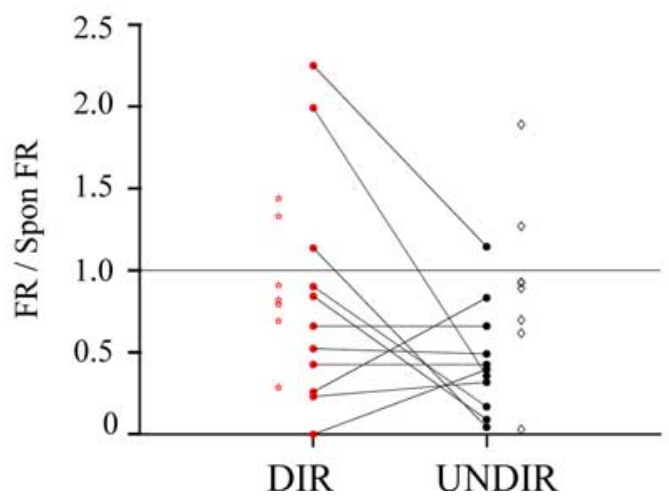

Figure 11. Firing rate was consistently suppressed after song termination in the UNDIR condition but not during the DIR condition. $A, B$, Raster plot of the activity of two LMAN neurons during an epoch from $200 \mathrm{~ms}$ before song termination to $2000 \mathrm{~ms}$ following song termination when the female was present (top) and when the male was alone (bottom). Spike trains are aligned by the offset of the last song syllable (blue lines). Activity was suppressed following song termination when the male was alone but not when the female was present. C, The mean normalized firing rate \pm SEM for 11 neurons (in six birds) for which there were both directed (red) and undirected (black) song bouts followed by at least $3 \mathrm{~s}$ of silence. Gray line $(y=1)$ indicates a firing rate equal to the average firing rate during nonsinging periods. $\boldsymbol{D}$, In the undirected condition, LMAN activity was consistently inhibited during the epoch $100-600 \mathrm{~ms}$ following song termination (indicated by curly brackets in $\boldsymbol{A}-\boldsymbol{C}$ ) compared with background nonsinging levels ( $p<0.005$, paired sign test).

in AFP activity could derive from network circuit properties. In the visual system, recurrent connections from cortex to thalamus shift the burst probability of thalamic relay neurons (for review, see Krahe and Gabbiani, 2004). In songbirds, recurrent connections are present in the AFP; LMAN neurons that project to RA also project to Area X, creating a feedback loop (Fig. 1A) (Nixdorf-Bergweiler et al., 1995; Vates and Nottebohm, 1995). One intriguing but unresolved question is whether this recurrent loop through Area X gates the firing mode in the AFP by modulating its afferent input.

Finally, context-dependent differences in AFP activity may merely reflect socially driven differences in afferent input from HVC. Previous studies of HVC neurons that project to Area X have been limited to a single behavioral condition (Kozhevnikov and Fee, 2007), so it is not known whether these cells exhibit social context-dependent differences in their firing properties.

Regardless of the mechanism by which social context regulates the firing mode of LMAN neurons, this study demonstrates a striking correspondence between behavioral context and firing properties of single neurons in a circuit required for vocal plasticity. More generally, our findings suggest that pallial-basal ganglia circuits contribute to motor learning and performance by actively regulating variability in motor output and by providing signals to bias motor output toward desired targets. Moreover, behavioral context can dramatically influence the degree to which such signals are present.

\section{References}

Balkowiec A, Katz DM (2000) Activity-dependent release of endogenous brain-derived neurotrophic factor from primary sensory neurons detected by ELISA in situ. J Neurosci 20:7417-7423.

Barnes TD, Kubota Y, Hu D, Jin DZ, Graybiel AM (2005) Activity of striatal neurons reflects dynamic encoding and recoding of procedural memories. Nature 437:1158-1161.

Bastian J, Nguyenkim J (2001) Dendritic modulation of burst-like firing in sensory neurons. J Neurophysiol 85:10-22.

Berry MJ, Warland DK, Meister M (1997) The structure and precision of retinal spike trains. Proc Natl Acad Sci U S A 94:5411-5416.

Billimoria CP, DiCaprio RA, Birmingham JT, Abbott LF, Marder E (2006) Neuromodulation of spike-timing precision in sensory neurons. J Neurosci 26:5910-5919.

Bottjer SW, Miesner EA, Arnold AP (1984) Forebrain lesions disrupt development but not maintenance of song in passerine birds. Science 224:901-903.

Bottjer SW, Halsema KA, Brown SA, Miesner EA (1989) Axonal connections of a forebrain nucleus involved with vocal learning in zebra finches. J Comp Neurol 279:312-326.

Brainard MS, Doupe AJ (2000) Interruption of a basal ganglia-forebrain circuit prevents plasticity of learned vocalizations. Nature 404:762-766.

Castelino CB, Ball GF (2005) A role for norepinephrine in the regulation of context-dependent ZENK expression in male zebra finches (Taeniopygia guttata). Eur J Neurosci 21:1962-1972.

Chi Z, Margoliash D (2001) Temporal precision and temporal drift in brain and behavior of zebra finch song. Neuron 32:899-910.

Cooper BG, Goller F (2006) Physiological insights into the social-contextdependent changes in the rhythm of the song motor program. J Neurophysiol 95:3798-3809.

Costa RM (2007) Plastic corticostriatal circuits for action learning: what's dopamine got to do with it? Ann N Y Acad Sci 1104:172-191.

Dave AS, Yu AC, Margoliash D (1998) Behavioral state modulation of auditory activity in a vocal motor system. Science 282:2250-2254.

Ding L, Perkel DJ (2002) Dopamine modulates excitability of spiny neurons in the avian basal ganglia. J Neurosci 22:5120-5218.

Ding L, Perkel DJ, Farries MA (2003) Presynaptic depression of glutamatergic synaptic transmission by D1-like dopamine receptor activation in the avian basal ganglia. J Neurosci 23:6086-6095.

Doupe AJ (1997) Song- and order-selective neurons in the songbird anterior forebrain and their emergence during vocal development. J Neurosci 17:1147-1167.

Doya K, Sejnowski TJ (1998) A computational model of birdsong learning by auditory experience and auditory feedback. In: Central auditory processing and neural modeling (Poon PWF, Brugge JF, eds), pp 77-88. New York: Plenum.

Doya K, Sejnowski TJ (2000) A computational model of avian song learn- 
ing. In: The new cognitive neurosciences (Gazzaniga MS, ed), pp 469482. Cambridge, MA: MIT.

Fiete IR, Fee MS, Seung HS (2007) Model of birdsong learning based on gradient estimation by dynamic perturbation of neural conductances. J Neurophysiol 98:2038-2057.

Glickstein M, Stein J (1991) Paradoxical movement in Parkinson's disease. Trends Neurosci 14:480-482.

Hahnloser RH, Kozhevnilov AA, Fee MS (2002) An ultra-sparse code underlies the generation of neural sequences in a songbird. Nature 419:65-70.

Hessler NA, Doupe AJ (1999a) Social context modulates singing-related neural activity in the songbird forebrain. Nat Neurosci 2:209-211.

Hessler NA, Doupe AJ (1999b) Singing-related neural activity in a dorsal forebrain-basal ganglia circuit of adult zebra finches. J Neurosci 19:10461-10481.

Hikosaka O, Nakamura K, Sakai K, Nakahara H (2002) Central mechanisms of motor skill learning. Curr Opin Neurobiol 12:217-222.

Hikosaka O, Nakamura K, Nakahara H (2006) Basal ganglia orient eyes to reward. J Neurophysiol 95:567-584.

Jarvis ED, Scharff C, Grossman MR, Ramos JA, Nottebohm F (1998) For whom the bird sings: context-dependent gene expression. Neuron 21:775-788.

Johnson F, Sablan MM, Bottjer SW (1995) Topographic organization of a forebrain pathway involved with vocal learning in zebra finches. J Comp Neurol 358:260-278.

Johnson F, Hohmann SE, DiStefano PS, Bottjer SW (1997) Neurotrophins suppress apoptosis induced by deafferentation of an avian motor-cortical region. J Neurosci 17:2101-2111.

Kao MH, Brainard MS (2006) Lesions of an avian basal ganglia circuit prevent context-dependent changes to song variability. J Neurophysiol 96:1441-1455.

Kao MH, Doupe AJ, Brainard MS (2005) Contributions of an avian basalganglia forebrain circuit to real-time modulation of song. Nature 433:638-643

Kara P, Reinagel P, Reid RC (2000) Low response variability in simultaneously recorded retinal, thamalic, and cortical neurons. Neuron 27:635-646.

Kargo WJ, Nitz DA (2004) Improvements in the signal-to-noise ratio of motor cortex cells distinguish early versus late phases of motor skill learning. J Neurosci 24:5560-5569.

Khumbani RD, Nolt MJ, Palmer LA (2007) Precision, reliability, and information-theoretic analysis of visual thalamocortical neurons. J Neurophys 98:2647-2663.

Kittelberger JM, Mooney R (1999) Lesions of an avian forebrain nucleus that disrupt song development alter synaptic connectivity and transmission in the vocal premotor pathway. J Neurosci 19:9385-9398.

Kittelberger JM, Mooney R (2005) Acute injections of brain-derived neurotrophic factor in a vocal premotor nucleus reversibly disrupt adult birdsong stability and trigger syllable deletion. J Neurobiol 62:406-424.

Kozhevnikov AA, Fee MS (2007) Singing-related activity of identified HVC neurons in the zebra finch. J Neurophysiol 97:4271-4283.

Krahe R, Gabbiani F (2004) Burst firing in sensory systems. Nat Rev Neurosci 5:13-23.

Lee IH, Assad JA (2003) Putaminal activity for simple reactions of selftimed movements. J Neurophysiol 89:2528-2537.

Leonardo A (2004) Experimental test of the birdsong error-correction model. Proc Natl Acad Sci U S A 101:16935-16940.

Leonardo A, Fee MS (2005) Ensemble coding of vocal control in birdsong. J Neurosci 25:652-661.

Lewicki MS (1994) Bayesian modelling and classification of neural signals. Neural Comput 6:1005-1030.

Lewis JW, Ryan SM, Arnold AP, Butcher LL (1981) Evidence for a catecholaminergic projection to area $\mathrm{X}$ in the zebra finch. J Comp Neurol 196:347-354

Lisman JE (1997) Bursts as a unit of neural information: making unreliable synapses reliable. Trends Neurosci 20:38-43.

Livingston FS, Mooney R (1997) Development of intrinsic and synaptic properties in a forebrain nucleus essential to avian song learning. J Neurosci 17:8997-9009.
Luo M, Ding L, Perkel DJ (2001) An avian basal ganglia pathway essential for vocal learning forms a closed topographic loop. J Neurosci 21:6836-6845.

Mainen ZF, Sejnowski TJ (1995) Reliability of spike timing in neocortical neurons. Science 268:1503-1506.

Marder E, Bucher D (2007) Understanding circuit dynamics using the stomatogastric nervous systems of lobsters and crabs. Annu Rev Physiol 69:291-316.

McCasland JS, Konishi M (1987) Neuronal control of bird song production. J Neurosci 7:23-39.

Merrill EG, Ainsworth A (1972) Glass-coated platinum-plated tungsten microelectrodes. Med Biol Eng 10:662-672.

Miller EK (2000) The prefrontal cortex and cognitive control. Nat Rev Neurosci 1:59-65.

Mooney R, Konishi M (1991) Two distinct inputs to an avian song nucleus activate different glutamate receptor subtypes on individual neurons. Proc Natl Acad Sci U S A 88:4075-4079.

Nelson DA, Marler P (1994) Selection-based learning in bird song development. Proc Natl Acad Sci U S A 91:10498-10501.

Nixdorf-Bergweiler BE, Lips MB, Heinemann U (1995) Electrophysiological and morphological evidence for a new projection of LMAN-neurones towards area X. Neuroreport 6:1729-1732.

Olveczky BP, Andalman AS, Fee MS (2005) Vocal experimentation in the juvenile songbird requires a basal ganglia circuit. PLoS Biol 3:e153.

Reinagel P, Reid RC (2002) Precise firing events are conserved across neurons. J Neurosci 22:6837-6841.

Rosen MJ, Mooney R (2000) Intrinsic and extrinsic contributions to auditory selectivity in a song nucleus critical for vocal plasticity. J Neurosci 20:5437-5448.

Sasaki A, Sotnikova TD, Gainetdinov RR, Jarvis ED (2006) Social contextdependent singing-regulated dopamine. J Neurosci 26:9010-9014.

Scharff C, Nottebohm F (1991) A comparative study of the behavioral deficits following lesions of various parts of the zebra finch song system: implications for vocal learning. J Neurosci 11:2896-2913.

Schultz W, Romo R (1992) Role of primate basal ganglia and frontal cortex in the internal generation of movements. I. Preparatory activity in the anterior striatum. Exp Brain Res 91:363-384.

Sheng HZ, Fields RD, Nelson PG (1993) Specific regulation of immediate early genes by patterned neuronal activity. J Neurosci Res 35:459-467.

Sossinka R, Böhner J (1980) Song types in the zebra finch (Poephila guttata castanotis) Z Tierpsychol 53:123-132.

Stark LL, Perkel DJ (1999) Two-stage, input-specific synaptic maturation in a nucleus essential for vocal production in the zebra finch. J Neurosci 19:9107-9116.

Sutton RS, Barto AG (1998) Reinforcement learning: an introduction. Cambridge, MA: MIT.

Troyer TW, Doupe AJ (2000) An associational model of birdsong sensorimotor learning. II. Temporal hierarchies and the learning of song sequence. J Neurophysiol 84:1224-1239.

Tumer EC, Brainard MS (2007) Performance variability enables adaptive plasticity of 'crystallized' adult birdsong. Nature 450:1240-1244.

Turner RW, Plant JR, Maler L (1996) Oscillatory and burst discharge across electosensory topographic maps. J Neurophysiol 76:2364-2382.

Uzzell VJ, Chichilnisky EJ (2004) Precision of spike trains in primate retinal ganglion cells. J Neurophysiol 92:780-789.

Vates GE, Nottebohm F (1995) Feedback circuitry within a song-learning pathway. Proc Natl Acad Sci U S A 92:5139-5143.

West MJ, King AP (1988) Female visual displays affect the development of male song in the cowbird. Nature 334:244-246.

Williams H, Mehta N (1999) Changes in adult zebra finch song require a forebrain nucleus that is not necessary for song production. J Neurobiol $39: 14-28$

Woolley SC, Doupe AJ (2008) Social context-induced song variation affects female behavior and gene expression. PLoS Biol 6:e62.

Worley PF, Bhat RV, Baraban JM, Erickson CA, McNaughton BL, Barnes CA (1993) Thresholds for synaptic activation of transcription factors in hippocampus: correlation with long-term enhancement. J Neurosci 13:4776-4786. 